Review Article

\title{
A systematic review and meta-analysis on the effects of yoga on weight-related outcomes
}

\author{
Romy Lauche ${ }^{\mathrm{a}, \mathrm{b}}$, Jost Langhorst ${ }^{\mathrm{a}}$, Myeong Soo Lee ${ }^{\mathrm{c}}$, Gustav Dobos ${ }^{\mathrm{a}}$, Holger Cramer ${ }^{\mathrm{a}, \mathrm{b}, *}$ \\ a Department of Internal and Integrative Medicine, Kliniken Essen-Mitte, Faculty of Medicine, University of Duisburg-Essen, Essen, Germany \\ ${ }^{\mathrm{b}}$ Australian Research Centre in Complementary and Integrative Medicine (ARCCIM), University of Technology Sydney, Sydney, Australia \\ c Medical Research Division, Korea Institute of Oriental Medicine, Daejeon, South Korea
}

\section{A R T I C L E I N F O}

\section{Article history:}

Received 10 September 2015

Received in revised form 26 January 2016

Accepted 17 March 2016

Available online 4 April 2016

\section{Keywords:}

Yoga

Complementary therapies

Body weight

Overweight

Obesity

\begin{abstract}
A B S T R A C T
Introduction. Overweight and obesity are among the most important modifiable risk factors for chronic diseases and premature death. The aim of this review was to systematically assess and analyze the effects of yoga on weight-related outcomes.

Methods. Medline/PubMed, Scopus, and the Cochrane Library were screened through March 2015 for randomized controlled trials on yoga for weight-related outcomes in the general population or overweight/obese individuals. Risk of bias was assessed using the Cochrane risk of bias tool on the following domains: selection bias, performance bias, detection bias, attrition bias, reporting bias, and other bias.

Results. Out of 445 records identified during literature search, 30 trials with a total of 2173 participants were included. No effects on weight, body mass index, body fat percentage or waist circumference were found. In studies with healthy adult participants an effect of yoga compared to usual care was found regarding waist/hip ratio (SMD =- $-1.00 ; 95 \% \mathrm{CI}=--1.44,-0.55 ; \mathrm{p}<0.001)$. In studies with overweight/obese participants only, effects relative to usual care were found for body mass index ( $\mathrm{SMD}=-0.99 ; 95 \% \mathrm{CI}=-1.67,-0.31 ; \mathrm{p}=$ 0.004). Effects however were not robust against selection bias; and publication bias could not be ruled out. No intervention-related adverse events were reported.

Conclusions. Despite methodological drawbacks, yoga can be preliminarily considered a safe and effective intervention to reduce body mass index in overweight or obese individuals.
\end{abstract}

(c) 2016 Elsevier Inc. All rights reserved.

\section{Contents}

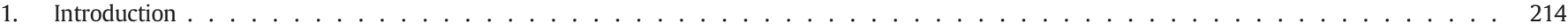

2. Methods . . . . . . . . . . . . . . . . . . . . . . . . . . . . . . . . . . . . . . . . . . . . . . . . . . . 214

2.1. Eligibility criteria . . . . . . . . . . . . . . . . . . . . . . . . . . . . . 214

2.1.1. Types of studies . . . . . . . . . . . . . . . . . . . . . . . . . . . . . . . . . . . . . . . . . . . . 214

2.1.2. Types of participants . . . . . . . . . . . . . . . . . . . . . . . . . . . . . . 214

2.1.3. Types of interventions . . . . . . . . . . . . . . . . . . . . . . . . . . . . . . . . . . . . . . . . . . . . . . . 214

2.1.4. Types of outcome measures . . . . . . . . . . . . . . . . . . . . . . . . . . . . . . . . . . 215

2.2. Search methods . . . . . . . . . . . . . . . . . . . . . . . . . . . . . . . . . . . . . 215

2.3. Data extraction and management . . . . . . . . . . . . . . . . . . . . . . . . . . . . . . . . . . . . . . . . . 215

2.4. Assessment of risk of bias in individual studies . . . . . . . . . . . . . . . . . . . . . . . . . . . . . . . . 215

2.5. Data analysis . . . . . . . . . . . . . . . . . . . . . . . . . . . . . . . . . . . . . . . . . . . . . 215

2.5.1. Assessment of overall effect size . . . . . . . . . . . . . . . . . . . . . . . . . 215

2.5.2. Assessment of heterogeneity . . . . . . . . . . . . . . . . . . . . . . . . . . . . . . . . . 215

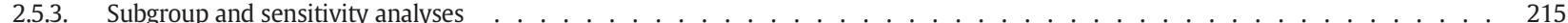

2.6. Risk of bias across studies . . . . . . . . . . . . . . . . . . . . . . . . . . . . . . . . . . . . . . . . 215

3. Results ... 215

3.1. Literature search . . . . . . . . . . . . . . . . . . . . . . . . . . . . . . . . . . . . 215

3.2. Study characteristics . . . . . . . . . . . . . . . . . . . . . . . . . . . . . . . . . 216

\footnotetext{
* Corresponding author at: Kliniken Essen-Mitte, Klinik für Naturheilkunde und Integrative Medizin, Knappschafts-Krankenhaus, Am Deimelsberg 34a, 45276 Essen, Germany. E-mail address: h.cramer@kliniken-essen-mitte.de (H. Cramer).
} 


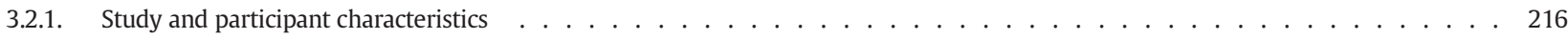

3.2.2. Intervention characteristics . . . . . . . . . . . . . . . . . . . . . . . . . . . . . . . . . . 217

3.2.3. Outcome measures . . . . . . . . . . . . . . . . . . . . . . . . . . . . 217

3.3. Risk of bias in individual studies . . . . . . . . . . . . . . . . . . . . . . . . . . . . . . . . . . . . 217

3.4. Analysis of overall effect . . . . . . . . . . . . . . . . . . . . . . . . . . . . . 218

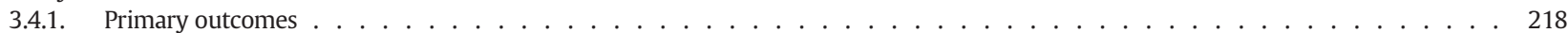

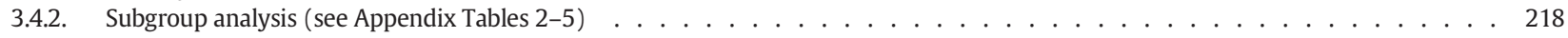

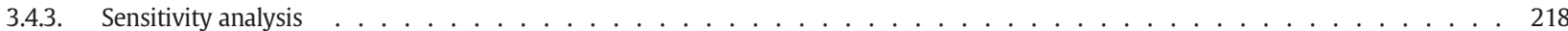

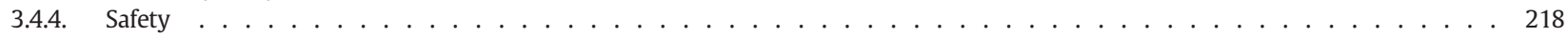

3.4.5. Risk of bias across studies . . . . . . . . . . . . . . . . . . . . . . . . . . . . . . . . . 218

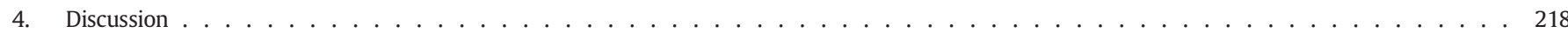

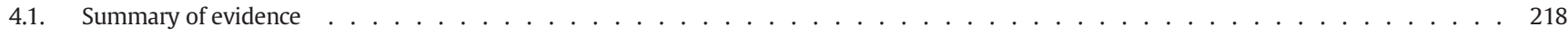

4.2. Agreements with prior systematic reviews . . . . . . . . . . . . . . . . . . . . . . . . . . . . . . . . . . . . . 218

4.3. Strengths and weaknesses . . . . . . . . . . . . . . . . . . . . . . . . . . . . . . . . . 219

4.4. How the intervention might work . . . . . . . . . . . . . . . . . . . . . . . . . . . . . . . . . . . 219

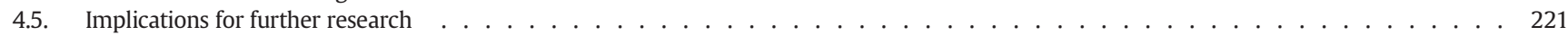

4.6. Implications for clinical practice . . . . . . . . . . . . . . . . . . . . . . . . . . . . . . . . . . . . 221

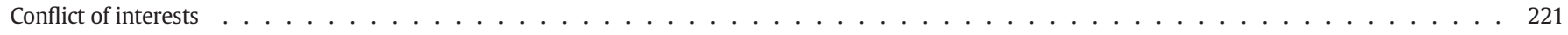

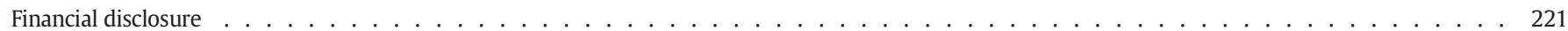

Appendix A. Complete search strategy for PubMed/Medline . . . . . . . . . . . . . . . . . . . . . . . . . . . . . . . . . . 221

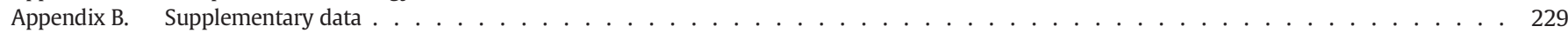

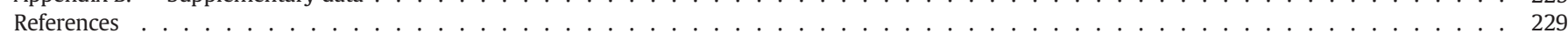

\section{Introduction}

Overweight and obesity are among the most important modifiable risk factors for chronic diseases and premature death (World Health Organization, 2014). About $69 \%$ of the US population are overweight or obese (National Center for Health Statistics, 2015). Worldwide the point prevalence is $39 \%$; with increasing rates especially in lowand middle-income countries (World Health Organization, 2014). Besides inadequate diet, the most important contributing factor to overweight and obesity is a sedentary lifestyle (Cecchini et al., 2010). Thus, regular physical activity is recommended in medical guidelines as the most important treatment option in non-morbid overweight or obesity; as well as a preventive intervention (American College of Cardiology/American Heart Association Task Force on Practice Guidelines - Obesity Expert Panel 2013, 2014; National Health and Medical Research Council, 2013). Given that a considerable number of individuals with weight problems are not adherent to recommended exercise regimens (Castellani et al., 2003), the investigation of alternative forms of exercise for weightrelated outcomes seems warranted.

One such alternative form of physical activity that is increasingly used for health purposes is yoga (Feuerstein, 1998; Iyengar, 1966). Yoga is most often associated with physical postures ('Asana'), breath control ('Pranayama'), and meditation ('Dhyana') in North America and Europe (Feuerstein, 1998; De Michelis, 2005); and is gaining increased popularity as a therapeutic method for various health issues. About 14 million adult Americans (6.1\% of the population) reported that yoga had been recommended to them by a physician or therapist (Macy, 2008). Indeed, about $80 \%$ of American yoga practitioners (more than 16 million people) reported that they had started practice explicitly to improve their health (Birdee et al., 2008; Clarke et al., 2002; Cramer et al., 2015a); and weight control is among the most frequently stated reasons for starting to practice (Park et al., 2014). While there is evidence to suggest that yoga is effective in promoting weight loss and improving body composition (Rioux and Ritenbaugh, 2013), no systematic review and/or metaanalysis on yoga for weight-related outcomes is available to date. Thus, the aim of this review was to systematically assess and analyze the effects of yoga on weight-related outcomes in the general population and in overweight/obese individuals by means of a metaanalysis.

\section{Methods}

This review was conducted in accordance with the Preferred Reporting Items for Systematic Reviews and Meta-Analyses (PRISMA) guidelines (Moher et al., 2009) and recommendations of the Cochrane Collaboration (Higgins and Green, 2008).

\subsection{Eligibility criteria}

\subsubsection{Types of studies}

Randomized controlled trials (RCTs) and cluster-randomized trials were eligible. No language restrictions were applied.

\subsubsection{Types of participants}

Studies on

a) children or adolescents or

b) adults

\section{were included if participants were}

i) healthy or from the general population (i.e. not selected based on their health status or weight) or

ii) overweight or obese.

The different subgroups were compared in subgroup analyses (a vs. b; i vs. ii).

Studies were excluded if overweight or obesity were comorbidities of diseases investigated in the trials, for example in studies investigating obese patients with hypertension. However, studies on participants with disease risk factor constellations, which are not a disease in itself, such as metabolic syndrome, were eligible. Studies on pregnant women and patients with eating disorders (anorexia nervosa, bulimia, binge eating) were excluded.

\subsubsection{Types of interventions}

2.1.3.1. Experimental. Studies were eligible if they included at least one of the following yoga practices based on yoga theory:

i) yoga postures or sequences of yoga postures

ii) breath control, meditation, and/or

iii) lifestyle advice. 
No restrictions were made regarding the tradition of the yoga intervention, the length, frequency or duration of the programs. Studies on multimodal interventions including yoga among others were excluded. Studies allowing individual co-interventions were eligible.

\subsubsection{Control. Studies comparing yoga to}
i) usual care
ii) exercise
iii) or other active control interventions

were eligible, but studies with head to head comparisons of different yoga interventions without non-yoga control groups were excluded.

\subsubsection{Types of outcome measures}

To be eligible for inclusion, studies had to assess at least one primary weight-related outcome:
i) body weight
ii) body mass index
iii) body fat percentage
iv) waist circumference
v) waist-hip ratio.

Secondary outcomes included safety of the intervention, assessed as number of patients with adverse events (AEs).

\subsection{Search methods}

The search strategy comprised three electronic databases from their inception through March 09, 2015: Medline/PubMed, Scopus, and the Cochrane Central Register of Controlled Trials. The literature search was constructed around search terms for 1 "yoga" and 2 "weight" and adapted for each database as necessary. The complete search strategy for PubMed/Medline is shown in the appendix.

Additionally, reference lists of identified original articles or reviews; and the tables of contents of the International Journal of Yoga Therapy and the Journal of Yoga \& Physical Therapy were searched manually; and trials identified as randomized controlled trials in a bibliometric analysis were checked for outcomes (Cramer et al., 2014a). Two reviewers independently screened and selected abstracts; potentially eligible articles were read in full by two reviewers. Disagreements were resolved through discussion with a third reviewer until consensus was reached. If necessary, additional information was obtained from the authors of the primary study.

\subsection{Data extraction and management}

Two reviewers independently extracted data on patient characteristics (e.g. age, gender, ethnicity), interventions (e.g. yoga type, frequency, and duration), control interventions (e.g. type, frequency, duration), and outcomes (e.g. outcome measures, assessment time points) using an a priori developed data extraction form. Discrepancies were resolved by discussion with a third reviewer until consensus was reached.

\subsection{Assessment of risk of bias in individual studies}

Two reviewers independently assessed risk of bias using the Cochrane risk of bias tool (Higgins and Green, 2008). This tool assesses risk of bias on seven domains: random sequence generation, allocation concealment, blinding of participants and personnel, blinding of outcome assessment, incomplete outcome data, selective reporting, and other sources of bias. For each domain, risk of bias was assessed as low; unclear; or high risk of bias.
Discrepancies were discussed with a third reviewer until consensus was reached.

\subsection{Data analysis}

\subsubsection{Assessment of overall effect size}

Separate meta-analyses were conducted for comparisons of yoga to different control interventions. Meta-analyses were conducted using Review Manager 5 software (Version 5.2, The Nordic Cochrane Centre, Copenhagen) by random effects models if at least two studies assessing this specific outcome were available. Standardized mean differences (SMD) with 95\% confidence intervals (CI) were calculated as the difference in means between groups divided by the pooled standard deviation using Hedges' correction for small study samples (Higgins and Green, 2008). Where no standard deviations were available, they were calculated from standard errors, confidence intervals or t-values (Higgins and Green, 2008), or attempts were made to obtain the missing data from the trial authors directly.

For all outcomes a negative SMD (i.e. lower scores in the yoga group) were defined to indicate beneficial effects of yoga compared to the control intervention. If necessary, values were inverted (Higgins and Green, 2008). Cohen's categories were used to evaluate the magnitude of the overall effect size with SMD < 0.2: negligible; SMD = 0.2-0.5: small; SMD $=0.5-0.8$ : medium; and SMD $>0.8$ : large effect sizes (Cohen, 1988).

\subsubsection{Assessment of heterogeneity}

Statistical heterogeneity between studies was analyzed using the $\mathrm{I}^{2}$ statistics; a measure of how much variance between studies can be attributed to differences between studies rather than chance. The magnitude of heterogeneity was categorized as (1) $\mathrm{I}^{2}=0-24 \%$ : low heterogeneity; $\mathrm{I}^{2}=25-49 \%$ : moderate; $\mathrm{I}^{2}=50-74 \%$ : substantial; and $\mathrm{I}^{2}=$ 75-100\%: considerable (Higgins and Green, 2008; Higgins et al., 2003). The $\mathrm{Chi}^{2}$ test was used to assess whether differences in results are compatible with chance alone. Due to the low power of this test in cases where only a few studies or studies with low sample size are included, a P-value $\leq 0.10$ was regarded to indicate significant heterogeneity (Cohen, 1988).

\subsubsection{Subgroup and sensitivity analyses}

Subgroup analyses were performed for the age of participants (children/adolescents vs. adults) and their weight status (participants: not selected based on their weight vs. overweight/obese participants).

To test the robustness of significant results, sensitivity analyses were conducted for studies with high versus low risk of selection bias (random sequence generation and allocation concealment).

If present in the respective meta-analysis, subgroup and sensitivity analyses were also used to explore possible reasons for statistical heterogeneity.

\subsection{Risk of bias across studies}

If at least ten studies were included in a meta-analysis, publication bias was assessed by visual inspection of funnel plots generated using Review Manager software (Higgins and Green, 2008; Egger et al., 1997). Roughly symmetrical funnel plots were regarded to indicate low risk of publication bias; and asymmetrical funnel plots were regarded to indicate high risk of publication bias.

\section{Results}

\subsection{Literature search}

The literature search retrieved 435 non-duplicate records of which 158 full-texts were assessed for eligibility, and 31 of them were eligible (Bera and Rajapurkar, 1993; Blumenthal et al., 1989; Chen et al., 2008; 
Table 1

Excluded studies.

\begin{tabular}{|c|c|}
\hline Excluded study (reference) & Reason for exclusion \\
\hline Mclver (2010) & Commentary \\
\hline $\begin{array}{l}\text { Cheung et al. (2012); Corey et al. (2014); LaCroix et al. (2012); Sharma et al. (2013); Singh et al. (2011); Hoogbruin et al. (2012); Lai et al. } \\
\text { (2010); Trivedi and Mishra (2014) }\end{array}$ & Conference abstract \\
\hline Bock et al. (2010); Bock et al. (2014); Cheema et al. (2011); Cohen et al. (2013); Kandula et al. (2013) & Trial protocol \\
\hline Neumark-Sztainer et al. (2011); Flaherty (2014); Guarracino et al. (2006) & No clinical trial \\
\hline $\begin{array}{l}\text { Ades et al. (2003); Ankad et al. (2011); Ankad Roopa et al. (2011); Armstrong and Scott Smedley (2003); Bhutkar et al. (2011); Choudhary } \\
\text { and Mishra (2013); Hunter et al. (2013); Malhotra et al. (2005); Murthy et al. (2011); Nagarathna and Nagendra (1987); Raj et al. (2011); } \\
\text { Ramos-Jimenez et al. (2009); Sarvottam et al. (2013); Satyanarayana et al. (1992); Sivasankaran et al. (2006); Telles et al. (1993); Telles } \\
\text { et al. (2010); Thomley et al. (2011); Villien et al. (2005); Yadav et al. (2012); Yadav et al. (2014); Yang and James (2014) }\end{array}$ & Not controlled \\
\hline $\begin{array}{l}\text { Begum et al. (2012); Cajka et al. (2013); Čajka et al. (2012); Ce et al. (2015); Chatterjee and Mondal (2014); Chaya et al. (2006); Chaya } \\
\text { et al. (2008); Dhananjai et al. (2013); Goncalves et al. (2011); Halder et al. (2015); Hegde et al. (2011); Herur et al. (2011); Kim et al. } \\
\text { (2014); Krejci (2011); Kubo et al. (2011); Madanmohan et al. (2008); Malhotra et al. (2010); Maninder et al. (2013); Miles et al. (2013); } \\
\text { Santhi Sri et al. (2014); Singh et al. (2008); Sukhsohale and Phatak (2012); Tyagi et al. (2014); Narendran et al. (2005a); Narendran et al. } \\
\text { (2005b) }\end{array}$ & Not randomized \\
\hline Stein et al. (2014); Sprod et al. (2015); Emery and Blumenthal (1990) & Secondary analysis \\
\hline $\begin{array}{l}\text { Bernstein et al. (2014); Chu et al. (2014a); Cramer et al. (2014b); Hartley et al. (2014); Innes and Vincent (2007); Jayasinghe (2004); Liu } \\
\text { et al. (2014); Manchanda and Madan (2014); Patel et al. (2012) }\end{array}$ & Review \\
\hline Brady (2007) & $\begin{array}{l}\text { Not published in a } \\
\text { peer-reviewed journal }\end{array}$ \\
\hline $\begin{array}{l}\text { Ades et al. (2005); Manchanda et al. (2000); Pal et al. (2013); Pal et al. (2011); Raghuram et al. (2014); Cadmus-Bertram et al. (2013); } \\
\text { Littman et al. (2012); Carei et al. (2010); Mclver et al. (2009); Mitchell et al. (2007); Yurtkuran et al. (2007); Cade et al. (2010); } \\
\text { McCaffrey et al. (2005); Murugesan et al. (2000); Rahnama et al. (2011); Cheung et al. (2014); Ebnezar et al. (2012); Sharma et al. } \\
\text { (2015); Nidhi et al. (2012); Nidhi et al. (2013a); Nidhi et al. (2013b); Rakhshani et al. (2015); Innes and Selfe (2012); Ikai et al. (2013); } \\
\text { Amita et al. (2009); Gordon et al. (2008); Nishanth et al. (2011); Shantakumari et al. (2013); Skoro-Kondza et al. (2009); Visweswaraiah } \\
\text { and Telles (2004) }\end{array}$ & Involved diseased participants \\
\hline $\begin{array}{l}\text { Dandekar (2013); Göring et al. (2013); Jorrakate et al. (2015); Rajajeyakumar et al. (2014); Santaella et al. (2011); Schmidt et al. (1997); } \\
\text { Sharma et al. (2014); Blumenthal et al. (1991); Elavsky and McAuley (2007b); Elavsky and McAuley (2007c) }\end{array}$ & No weight-related outcome \\
\hline $\begin{array}{l}\text { Arciero et al. (2014); Boxer et al. (2010); Bryan and Zipp (2014); DeBar et al. (2012); Kenny et al. (2010); Kim et al. (2008); Park et al. } \\
\text { (2010); Ramen et al. (2013); Sabet Sarvestani et al. (2009); Ziv et al. (2013) }\end{array}$ & No yoga \\
\hline
\end{tabular}

Chen et al., 2010; Cohen et al., 2008a; DiPietro et al., 1998; Elavsky and McAuley, 2007a; Harbans et al., 2011; Hegde et al., 2013; Kanaya et al., 2014; Kanojia et al., 2013; Lee et al., 2012; Lu and Wang, 2007; Mahajan et al., 1999; Manjunath and Telles, 2012; McDermott et al., 2014; Mooventhan and Khode, 2014; Ray et al., 2001; Sakuma et al., 2012; Seo et al., 2012; Shukla and Gehlot, 2014; Stachenfeld et al., 1998; Telles et al., 2014; Telles et al., 2013; Thiyagarajan et al., 2015; Tracy and Hart, 2013; Van Puymbroeck et al., 2007; Yang et al., 2011; Khatri et al., 2007; Kim et al., 2013; Manchanda et al., 2013), while 127 of those full-texts were excluded (Table 1) (McIver, 2010; Cheung et al., 2012; Corey et al., 2014; LaCroix et al., 2012; Sharma et al., 2013; Singh et al., 2011; Hoogbruin et al., 2012; Lai et al., 2010; Trivedi and Mishra, 2014; Bock et al., 2010; Bock et al., 2014; Cheema et al., 2011; Cohen et al., 2013; Kandula et al., 2013; Neumark-Sztainer et al., 2011; Flaherty, 2014; Guarracino et al., 2006; Ades et al., 2003; Ankad et al., 2011; Ankad Roopa et al., 2011; Armstrong and Scott Smedley, 2003; Bhutkar et al., 2011; Choudhary and Mishra, 2013; Hunter et al., 2013; Malhotra et al., 2005; Murthy et al., 2011; Nagarathna and Nagendra, 1987; Raj et al., 2011; Ramos-Jimenez et al., 2009; Sarvottam et al., 2013; Satyanarayana et al., 1992; Sivasankaran et al., 2006; Telles et al., 1993; Telles et al., 2010; Thomley et al., 2011; Villien et al., 2005; Yadav et al., 2012; Yadav et al., 2014; Yang and James, 2014; Begum et al., 2012; Cajka et al., 2013; Čajka et al., 2012; Ce et al., 2015; Chatterjee and Mondal, 2014; Chaya et al., 2006; Chaya et al., 2008; Dhananjai et al., 2013; Goncalves et al., 2011; Halder et al., 2015; Hegde et al., 2011; Herur et al., 2011; Kim et al., 2014; Krejci, 2011; Kubo et al., 2011; Madanmohan et al., 2008; Malhotra et al., 2010; Maninder et al., 2013; Miles et al., 2013; Santhi Sri et al., 2014; Singh et al., 2008; Sukhsohale and Phatak, 2012; Tyagi et al., 2014; Narendran et al., 2005a; Narendran et al., 2005b; Stein et al., 2014; Sprod et al., 2015; Emery and Blumenthal, 1990; Bar et al., 2014; Chu et al., 2014a; Cramer et al., 2014b; Hartley et al., 2014; Innes and Vincent, 2007; Jayasinghe, 2004; Liu et al., 2014; Manchanda and Madan, 2014; Patel et al., 2012; Brady, 2007; Ades et al., 2005; Manchanda et al., 2000; Pal et al., 2013; Pal et al., 2011; Raghuram et al., 2014; Cadmus-Bertram et al., 2013; Littman et al., 2012; Carei et al., 2010; Mclver et al., 2009; Mitchell et al., 2007; Yurtkuran et al., 2007; Cade et al., 2010; McCaffrey et al., 2005; Murugesan et al., 2000; Rahnama et al., 2011; Cheung et al., 2014; Ebnezar et al., 2012; Sharma et al., 2015; Nidhi et al., 2012; Nidhi et al., 2013a; Nidhi et al., 2013b; Rakhshani et al., 2015; Innes and Selfe, 2012; Ikai et al., 2013; Amita et al., 2009; Gordon et al., 2008; Nishanth et al., 2011; Shantakumari et al., 2013; Skoro-Kondza et al., 2009; Visweswaraiah and Telles, 2004; Dandekar, 2013; Göring et al., 2013; Jorrakate et al., 2015; Rajajeyakumar et al., 2014; Santaella et al., 2011; Schmidt et al., 1997; Sharma et al., 2014; Blumenthal et al., 1991; Elavsky and McAuley, 2007b; Elavsky and McAuley, 2007c; Arciero et al., 2014; Boxer et al., 2010; Bryan and Zipp, 2014; DeBar et al., 2012; Kenny et al., 2010; Kim et al., 2008; Park et al., 2010; Ramen et al., 2013; Sabet Sarvestani et al., 2009; Ziv et al., 2013).

Thirty-one full-text articles reporting on 30 RCTs involving a total of 2173 participants were included in the qualitative analysis; and 27 articles on 26 RCTs were meta-analyzed (Fig. 1). All but one article (published in Chinese) (Lu and Wang, 2007) were published in English.

\subsection{Study characteristics}

Characteristics of the samples, interventions, outcome assessments, and results are shown in Appendix Table 1.

\subsubsection{Study and participant characteristics}

Of the 30 studies that were included, 15 originated from India (Bera and Rajapurkar, 1993; Harbans et al., 2011; Hegde et al., 2013; Kanojia et al., 2013; Mahajan et al., 1999; Manjunath and Telles, 2012; McDermott et al., 2014; Mooventhan and Khode, 2014; Ray et al., 2001; Shukla and Gehlot, 2014; Telles et al., 2014; Telles et al., 2013; Thiyagarajan et al., 2015; Khatri et al., 2007; Manchanda et al., 2013), 8 from the USA (Blumenthal et al., 1989; Cohen et al., 2008a; Elavsky and McAuley, 2007a; Kanaya et al., 2014; Stachenfeld et al., 1998; Tracy and Hart, 2013; Van Puymbroeck et al., 2007; Yang et al., 2011), 


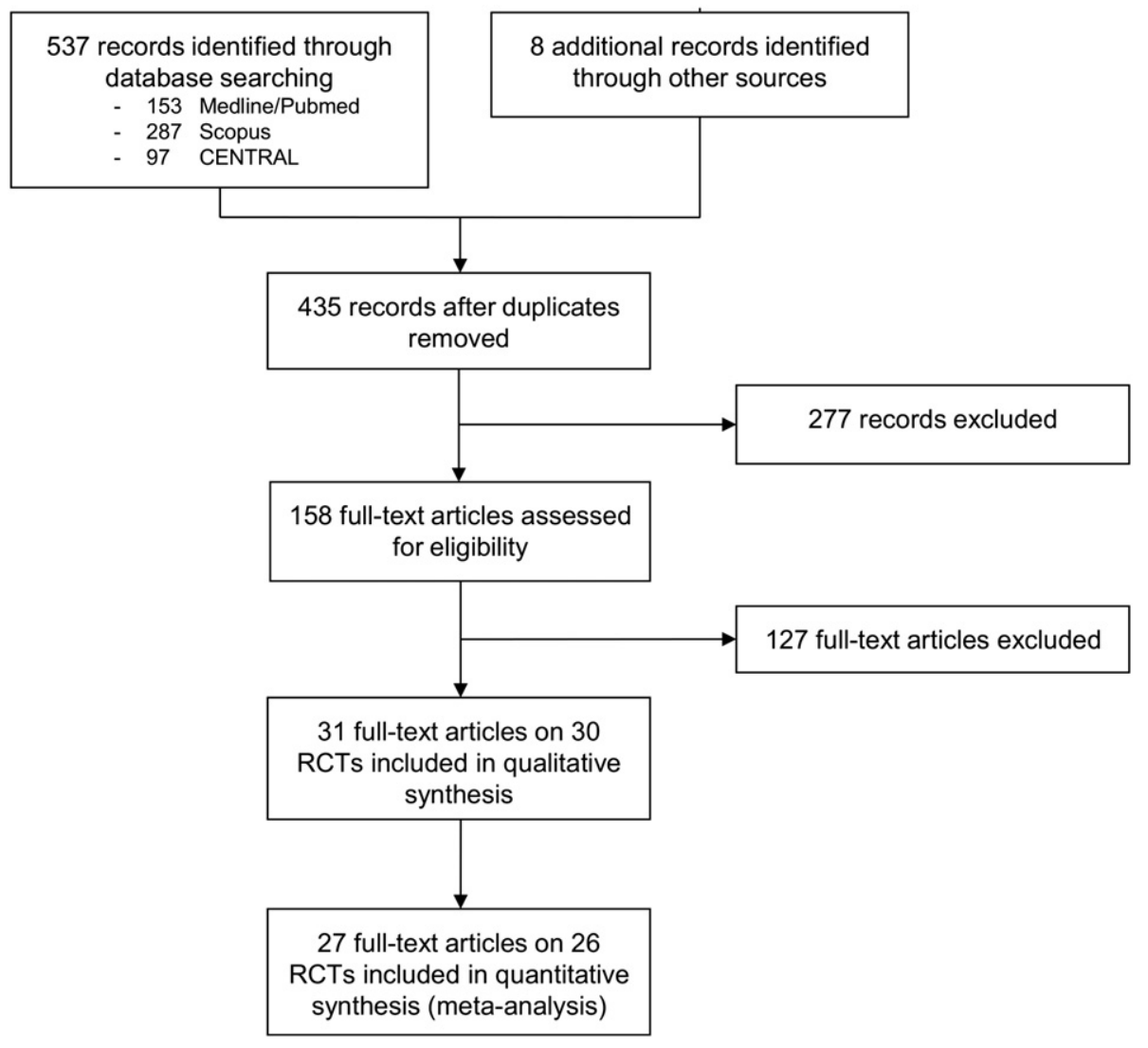

Fig. 1. Flowchart of the results of the literature search.

3 from Korea (Lee et al., 2012; Seo et al., 2012; Kim et al., 2013), 2 from Taiwan (Chen et al., 2008; Chen et al., 2010), and one each from Japan (Sakuma et al., 2012) and China (Lu and Wang, 2007). Ten of the trials explicitly investigated overweight or obese individuals (Cohen et al., 2008a; Harbans et al., 2011; Kanaya et al., 2014; Lee et al., 2012; Seo et al., 2012; Shukla and Gehlot, 2014; Telles et al., 2014; Khatri et al., 2007; Kim et al., 2013; Manchanda et al., 2013); and three trials were conducted on children/adolescents (Bera and Rajapurkar, 1993; Seo et al., 2012; Telles et al., 2013).

The sample size ranged from 17 to 204 with a median of 60 . Participant's mean age ranged from 10.4 to 75.4 years with a median of 50.0 years. A median of $48.3 \%$ of participants was female.

\subsubsection{Intervention characteristics}

Two studies each used Silver yoga (Chen et al., 2008; Chen et al., 2010) and Hatha Yoga (Ray et al., 2001; Kim et al., 2013), one each used Restorative Yoga (Kanaya et al., 2014) Yogasana,(Sakuma et al., 2012), Bikram (Tracy and Hart, 2013), Vinyasa Yoga (Yang et al., 2011), and yogic lifestyle intervention (Mahajan et al., 1999), and 21 did not report the yoga style used (Appendix Table 1). Twenty-six studies reported using yoga postures while one used only breathing exercises; and the majority of trials also utilized breathing, meditation or relaxation exercises. The duration of yoga programs ranged from 2 to 52 weeks with a median of 12 weeks; participants practiced yoga on 1-7 days per week (median: 3.5) with session of 10-90 (median: 60) minutes length. Intervention adherence was reported by only nine studies (Blumenthal et al., 1989; Chen et al., 2008; Elavsky and McAuley, 2007a; Hegde et al., 2013; Kanaya et al., 2014; McDermott et al., 2014; Manchanda et al., 2013; Cohen et al., 2008b; Yang et al., 2011); participants in those studies attended a mean of 63.0\%-95.8\% (median: $78.2 \%$ ) of the prescribed yoga sessions. Regarding control interventions, nineteen studies compared yoga to usual care or no specific treatment, eight studies compared yoga to exercise, three studies compared yoga to lifestyle modification (Mahajan et al., 1999; McDermott et al., 2014; Thiyagarajan et al., 2015); and one trial compared yoga combined with diet to diet alone (Shukla and Gehlot, 2014). One trial compared yoga to herbal medicines (Manjunath and Telles, 2012) and could therefore not be included in the meta-analysis. In addition to a yoga group and a non-yoga exercise control group, one trial included a combination of yoga and exercise as a third study arm (Lu and Wang, 2007). This third group was not included in the meta-analysis. Another trial compared two different yoga interventions with each other and with an untreated control group (Chen et al., 2008). In meta-analysis, the two yoga interventions could be combined into one group. In most exercise studies yoga and exercise interventions were matched for program length, frequency and duration of the sessions.

\subsubsection{Outcome measures}

All studies assessed outcomes immediately after the end of the intervention. Body weight was assessed in 22 studies, body mass index in 17 , body fat percentage in 9 , waist circumference in 15 and waist hip ratio in 6 studies. Safety was reported in five trials only.

\subsection{Risk of bias in individual studies}

Risk of bias in individual studies is shown in Table 2. Eleven studies had reported adequate random sequence generation, but only seven reported allocation concealment; none of the trials reported blinding of patients and personnel; but six studies reported adequate blinding of 
Table 2

Risk of Bias assessment.

\begin{tabular}{|c|c|c|c|c|c|c|c|}
\hline Reference & $\begin{array}{l}\text { Random sequence } \\
\text { generation } \\
\text { (selection bias) }\end{array}$ & $\begin{array}{l}\text { Allocation } \\
\text { concealment } \\
\text { (selection bias) }\end{array}$ & $\begin{array}{l}\text { Blinding of participants } \\
\text { and personnel } \\
\text { (performance bias) }\end{array}$ & $\begin{array}{l}\text { Blinding of } \\
\text { outcome assessment } \\
\text { (detection bias) }\end{array}$ & $\begin{array}{l}\text { Incomplete outcome } \\
\text { data (attrition bias) }\end{array}$ & $\begin{array}{l}\text { Selective reporting } \\
\text { (reporting bias) }\end{array}$ & Other bias \\
\hline Bera (1993) & unclear & unclear & unclear & unclear & unclear & unclear & low \\
\hline Blumenthal (1989) & unclear & unclear & unclear & unclear & low & high & low \\
\hline Chen (2008) & low & high & unclear & unclear & low & high & low \\
\hline Chen (2010) & unclear & high & unclear & unclear & low & high & high \\
\hline Cohen (2008) & unclear & high & high & unclear & low & low & high \\
\hline Elavsky (2007) & low & unclear & unclear & low & low & high & low \\
\hline Harbans (2011) & unclear & unclear & unclear & unclear & high & low & high \\
\hline Hegde (2013) & low & unclear & high & high & low & low & low \\
\hline Kim (2013) & low & low & unclear & low & low & low & low \\
\hline Kanaya (2014) & unclear & low & unclear & unclear & low & low & low \\
\hline Kanojia (2013) & unclear & unclear & unclear & unclear & unclear & low & low \\
\hline Khatri (2007) & unclear & unclear & unclear & unclear & unclear & high & high \\
\hline Lee $(2012)$ & unclear & unclear & unclear & unclear & low & low & low \\
\hline Lu (2007) & unclear & unclear & unclear & unclear & unclear & low & low \\
\hline Mahajan (1999) & unclear & unclear & unclear & unclear & unclear & low & low \\
\hline Manchanda (2013) & unclear & unclear & high & high & high & low & unclear \\
\hline Manjunath (2012) & low & low & unclear & unclear & low & High & high \\
\hline McDermott (2014) & low & low & high & low & low & low & low \\
\hline Mooventhan (2014) & low & low & unclear & unclear & low & low & low \\
\hline Ray (2001) & unclear & unclear & unclear & unclear & high & low & low \\
\hline Sakuma (2012) & low & low & unclear & unclear & high & High & high \\
\hline Shukla Ravi (2014) & unclear & unclear & unclear & unclear & unclear & unclear & high \\
\hline Seo (2012) & unclear & unclear & unclear & unclear & high & low & low \\
\hline Stachenfeld (1998) & unclear & unclear & unclear & unclear & unclear & low & high \\
\hline Telles (2013) & low & unclear & unclear & low & low & low & high \\
\hline Telles (2014) & low & unclear & unclear & unclear & high & unclear & high \\
\hline Thiyagarajan (2015) & low & low & high & high & high & low & high \\
\hline Tracy (2013) & unclear & unclear & unclear & unclear & high & unclear & high \\
\hline Van Puymbroeck (2007) & unclear & unclear & high & low & high & low & high \\
\hline Yang (2011) & unclear & unclear & unclear & low & low & low & low \\
\hline
\end{tabular}

outcome assessement; fourteen trials had low risk of attrition bias, and eighteen were free of suspected selective reporting.

\subsection{Analysis of overall effect}

\subsubsection{Primary outcomes}

Meta-analyses revealed no effects on weight (Fig. 2), BMI, body fat percentage or waist circumference for yoga compared to usual care, exercise or lifestyle modification. For waist hip ratio a significant effect was found for yoga compared to usual care (SMD $=-1.00 ; 95 \%$ $\mathrm{CI}=-1.44$ to $-0.55 ; \mathrm{P}<0.0001$ ), see Table 3 . One trial compared yoga to an herbal medicine compound but did not include statistical tests for group differences (Harbans et al., 2011).

\subsubsection{Subgroup analysis (see Appendix Tables 2-5)}

No effects were found for trials including children/adolescents only. Effects were the same in trials for adults only or for participants not selected for weight status only compared to the complete sample.

For studies on overweight or obese participants significant effects were found for body mass index only (SMD $=-0.99 ; 95 \%$ $\mathrm{CI}=-1.67$ to $-0.31 ; \mathrm{P}=0.004$ ) for yoga compared to usual care.

\subsubsection{Sensitivity analysis}

Due to the paucity of eligible trials, no sensitivity analyses could be conducted for studies with low risk of selection bias.

\subsubsection{Safety}

Only seven studies reported safety-related data, however they stated that no adverse events were reported (Cohen et al., 2008a; Sakuma et al., 2012), no adverse events during the intervention occurred (Telles et al., 2014; Van Puymbroeck et al., 2007), no clinically significant adverse events were reported or observed (Harbans et al., 2011), that no special concerns were found (Chen et al., 2008) or that no participant dropped out because of side effects (Kim et al., 2013).

\subsubsection{Risk of bias across studies}

Funnel plots were asymmetrical for weight, body mass index, and waist circumference; indicating high risk of publication bias (see Appendix Fig. 1).

\section{Discussion}

\subsection{Summary of evidence}

In this systematic review of 30 randomized controlled trials, only little evidence for effects of yoga on weight-related outcomes has been found. Significant changes in body mass index were identified only in studies with overweight/obese participants practicing yoga compared to usual care, while significant effects for waist hip ratio were found for yoga compared to usual care in adults who were not selected based on their weight only. Effects however were not robust against potential methodological bias and publication bias could not be ruled out. Furthermore safety was insufficiently reported.

\subsection{Agreements with prior systematic reviews}

Only one prior review explicitly investigated the effects of yoga on weight and related parameters. Rioux and Ritenbaugh (2013) conducted a narrative review, and included 17 trials that used yoga as an intervention for weight loss; among them uncontrolled, controlled and randomized controlled trials. Their risk assessment was based on a self-developed instrument. The authors concluded that therapeutic yoga was frequently effective in promotion weight loss or improving body composition in included trials. They also found that increased 


\begin{tabular}{|c|c|c|c|c|c|c|c|c|c|}
\hline \multirow{3}{*}{$\begin{array}{l}\text { Subgroups/Studies } \\
\text { Yoga vs. Usual Care }\end{array}$} & \multicolumn{3}{|c|}{ Yoga } & \multicolumn{2}{|c|}{ Control } & & \multirow{2}{*}{$\begin{array}{l}\text { Std. Mean Difference } \\
\text { IV, Random, } 95 \% \mathrm{Cl}\end{array}$} & \multirow{2}{*}{$\begin{array}{l}\text { Std. Mean Difference } \\
\text { IV, Random, } 95 \% \mathrm{CI}\end{array}$} \\
\hline & \multirow[t]{2}{*}{ Mean } & \multirow[t]{2}{*}{ SD 1} & \multirow[t]{2}{*}{ Total } & \multirow[t]{2}{*}{ Mean } & \multirow[t]{2}{*}{ SD 1} & & & & \\
\hline & & & & & & \multicolumn{2}{|c|}{ Total Weight } & \multirow{2}{*}{$0.49[-0.14,1.12]$} & \\
\hline Bera et al., 1993 & 44.81 & 9.39 & 20 & 40.28 & 8.65 & 20 & $8.4 \%$ & & $\square$ \\
\hline Blumenthal et al., 1989 & 0.6 & 2.7 & 34 & 0.3 & 1.7 & 34 & $9.3 \%$ & $0.13[-0.34,0.61]$ & \\
\hline Chen et al., 2008 & 58.97 & 9.39 & 110 & 61.77 & 8.65 & 66 & $10.1 \%$ & $-0.31[-0.61,0.00]$ & \\
\hline Cohen et al., 2008 & -0.1 & 2.7 & 12 & 2.2 & 9 & 12 & $7.3 \%$ & $-0.33[-1.14,0.47]$ & \\
\hline Kanojia et al., 2013 & 50.72 & 8.93 & 25 & 49.15 & 6.85 & 25 & $8.8 \%$ & $0.19[-0.36,0.75]$ & \\
\hline Lee et al., 2012 & 58.06 & 4.31 & 8 & 62.88 & 4.5 & 8 & $5.9 \%$ & $-1.03[-2.10,0.03]$ & \\
\hline Manchanda et al., 2013 & 86.91 & 12.53 & 34 & 89.14 & 13.55 & 43 & $9.4 \%$ & $-0.17[-0.62,0.28]$ & \\
\hline Mooventhan et al., 2014 & 57.52 & 12.18 & 40 & 51.97 & 11.08 & 39 & $9.4 \%$ & $0.47[0.02,0.92]$ & \\
\hline Sakuma et al., 2012 & 52.1 & 8 & 67 & 52.2 & 7.5 & 31 & $9.5 \%$ & $-0.01[-0.44,0.41]$ & \\
\hline Seo et al., 2012 & 74.49 & 7.06 & 10 & 78.13 & 10.44 & 10 & $6.8 \%$ & $-0.39[-1.28,0.50]$ & \\
\hline Shukla et al., 2014 & -5.2 & 1.48 & 30 & -2.16 & 1.02 & 30 & $8.1 \%$ & $-2.36[-3.03,-1.69]$ & \\
\hline $\begin{array}{l}\text { Yang et al., } 2011 \\
\text { Subtotal }(95 \% \mathrm{Cl})\end{array}$ & 79.24 & 13.52 & $\begin{array}{r}12 \\
402\end{array}$ & 85.91 & 13.34 & $\begin{array}{r}10 \\
328\end{array}$ & $\begin{array}{r}7.0 \% \\
100.0 \%\end{array}$ & $\begin{array}{l}-0.48[-1.33,0.38] \\
-0.27[-0.65,0.11]\end{array}$ & \\
\hline \multicolumn{10}{|c|}{ Heterogeneity: $\mathrm{Chi}^{2}=61.27, \mathrm{df}=11(\mathrm{P}<0.00001) ; \mathrm{I}^{2}=82 \%$} \\
\hline \multicolumn{10}{|l|}{ Yoga vs. Exercise } \\
\hline Blumenthal et al., 1989 & 0.6 & 2.7 & 34 & 0.5 & 1.7 & 32 & $24.3 \%$ & $0.04[-0.44,0.53]$ & \\
\hline Kanaya et al., 2014 & -1.3 & 2.83 & 88 & -0.7 & 3.21 & 83 & $37.7 \%$ & $-0.20[-0.50,0.10]$ & \\
\hline Lu et al., 2007 & 62.66 & 6.01 & 21 & 61.49 & 5.41 & 21 & $18.2 \%$ & $0.20[-0.41,0.81]$ & \\
\hline Ray et al., 2001 & 62.2 & 3.5 & 17 & 64.1 & 3.9 & 11 & $12.7 \%$ & $-0.50[-1.28,0.27]$ & \\
\hline $\begin{array}{l}\text { Stachenfeld et al., } 1998 \\
\text { Subtotal }(95 \% \mathrm{Cl})\end{array}$ & 64 & 4 & $\begin{array}{r}9 \\
169\end{array}$ & 69 & 4 & $\begin{array}{r}7 \\
154\end{array}$ & $\begin{array}{r}7.1 \% \\
100.0 \%\end{array}$ & $\begin{array}{l}-1.18[-2.28,-0.09] \\
-0.18[-0.49,0.14]\end{array}$ & \\
\hline \multicolumn{10}{|c|}{ Heterogeneity: $\mathrm{Chi}^{2}=6.21, \mathrm{df}=4(\mathrm{P}=0.18) ; \mathrm{I}^{2}=36 \%$} \\
\hline \multicolumn{10}{|c|}{ Test for overall effect: $Z=1.11(P=0.27)$} \\
\hline \multicolumn{10}{|l|}{ Yoga vs. Lifestyle } \\
\hline Mahajan et al., 1999 & 70.48 & 10.48 & 30 & 74.29 & 12.04 & 23 & $27.4 \%$ & $-0.34[-0.88,0.21]$ & \\
\hline McDermott et al., 2014 & -0.2 & 1.26 & 20 & 0.6 & 1.26 & 18 & $19.2 \%$ & $-0.62[-1.28,0.03]$ & \\
\hline $\begin{array}{l}\text { Thiagarayan et al., } 2015 \\
\text { Subtotal }(95 \% \mathrm{Cl})\end{array}$ & 567.12 & 9.5 & $\begin{array}{r}51 \\
101\end{array}$ & 68.34 & 11.52 & $\begin{array}{l}49 \\
90\end{array}$ & $\begin{array}{r}53.3 \% \\
100.0 \%\end{array}$ & $\begin{array}{l}-0.11[-0.51,0.28] \\
-0.27[-0.56,0.01]\end{array}$ & \\
\hline \multicolumn{10}{|c|}{ Heterogeneity: $\mathrm{Ch}^{2}=1.78, \mathrm{df}=2(\mathrm{P}=0.41) ; \mathrm{I}^{2}=0 \%$} \\
\hline \multicolumn{10}{|c|}{ Test for overall effect: $Z=1.87(P=0.06)$} \\
\hline & & & & & & & & & $\begin{array}{ll}1 & -1\end{array}$ \\
\hline Test for subgroup differe & ences: $\mathrm{C}$ & $\mathrm{Chi}^{2}=0$. & $.24, d$ & $=2(P$ & $0.89), I^{2}$ & $I^{2}=0 \%$ & & & Favours co \\
\hline
\end{tabular}

Fig. 2. Forest plot and effect sizes for yoga compared to usual care, exercise and lifestyle modification for the outcome weight.

frequency, longer duration, dietary components, residential components, a multi-component yoga and home practice increased the effectiveness.

In comparison our current review found only very limited evidence of yoga's efficacy for weight control in overweight/obese patients; however effects were not robust against bias. Subgroup analysis or meta-regression based on intensity, duration and yoga components could not be conducted due to heterogeneity of trials with very little overlap. The present review had also used the Cochrane risk of bias tool and found only a few trials with low risk of selection bias in general.

Another review on the effects of yoga on cardiovascular risk factors found effects of yoga on waist circumference and waist/hip ratio in high-risk groups but did not include other weight-related outcomes (Cramer et al., 2014b). Other reviews reported comparable effects (Chu et al., 2014b; Yang, 2007).

\subsection{Strengths and weaknesses}

To the best of our knowledge, this is the first systematic review and/ or meta-analysis available on yoga for weight management. Strengths of this review include the comprehensive literature search and the inclusion of yoga trials on the general population as well as overweight/ obese participants. The primary limitation of this review is the paucity of eligible studies especially for overweight/obese participants, rendering further subgroup analyses impossible. Another major limitation is the insufficient reporting and/or low methodological quality of the included studies, limiting the interpretability of the results. Intervention adherence was reported in less than one third of the included studies; thus the influence of adherence on study outcomes could not be evaluated. The inclusion of studies on metabolic syndrome can be regarded as a limitation because metabolic syndrome - although not defined as a disease - might nevertheless be associated with pronounced symptoms.

\subsection{How the intervention might work}

While yoga is often considered a form of exercise, beginner-level yoga sessions are not ordinarily considered sufficient for improving cardiovascular fitness (Hagins et al., 2007). More intensive forms of yoga however can contribute to higher energy expenditure (Hagins et al., 
Table 3

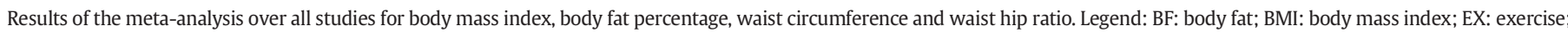
LSM: lifestyle modification; UC: usual care; WC: waist circumference; WHR: waist hip ratio.

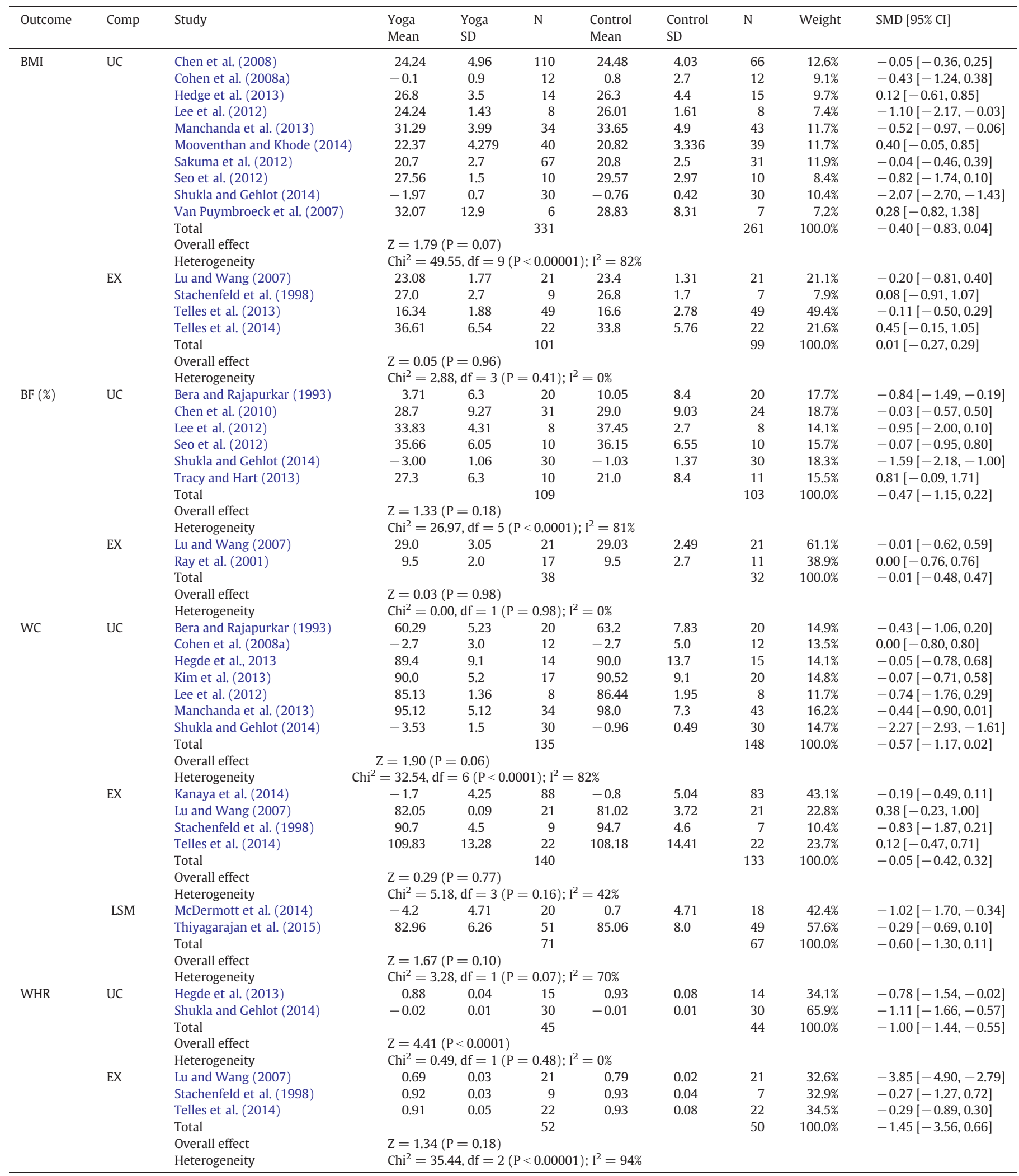

2007; Mody, 2011; Ray et al., 2011); and can thus contribute to weight loss and maintenance (Kristal et al., 2005). Moreover, yoga can ameliorate back and joint pain (Cramer et al., 2013a; Cramer et al., 2013b;
Haaz and Bartlett, 2011; Bussing et al., 2012), and increase levels of other, non-yoga physical activity (Bryan et al., 2012). Beyond exercise, yoga can also encompass yogic dietary advice; as well as relaxation, 
meditation, breath control and positive thinking. Thus, yoga has been shown to effectively decrease chronic depression (Cramer et al., 2013c) and stress (Chong et al., 2011); which might in turn reduce emotional overeating and resulting overweight (Dallman et al., 2003). This way, yoga interventions target both, physical and emotional maintaining factors of obesity.

\subsection{Implications for further research}

Given that the main drawback of this review was the insufficient reporting of trial methodology, authors of future research should improve the reporting of yoga trials and follow commonly accepted reporting guidelines (e.g. CONSORT) (Schulz et al., 2010). Further trials should ensure rigorous methodology such as a-priori sample size calculations to prevent the trial from being underpowered. They should further ensure adequate randomization, allocation concealment, intention-to-treat analysis, and blinding of at least outcome assessors (Schulz et al., 2010).

Only seven trials in this review explicitly investigated overweight/ obese samples, and two of them could not be included in the metaanalysis due to their unique control groups. The remaining trials when combined - only included 60 patients in the meta-analytic comparison of yoga and usual care. More trials on yoga for overweight or obese people therefore are warranted. Since overweight and obesity might also be associated with eating disorders, trials on for example binge eating are urgently needed, as such participants may require special consideration.

Future trials must also improve reporting of safety. Even though a systematic review of randomized controlled trials found no evidence for serious yoga-associated adverse events or an accumulation of adverse events compared to usual care or exercise (Cramer et al., 2015b), evidence was limited because only one third of the trials actually reported safety and most of them insufficiently. Yoga has also been occasionally associated with serious adverse events (Cramer et al., 2013d). Especially overweight and obese patients may be required to present a certain physical fitness; and certain poses might be associated with an increased risk of injuries. This should be an additional focus of further trials.

\subsection{Implications for clinical practice}

Based on the results of this meta-analysis, no recommendation can be made for or against yoga to influence weight-related outcomes in children and adolescents. Despite the methodological drawbacks of the included trials, yoga can however be preliminarily considered a safe and effective intervention to reduce body mass index in overweight or obese but otherwise healthy adults. It has been shown that while women and younger individuals are less adherent to physical activity interventions targeting overweight and obesity (Bautista-Castano et al., 2004), those individuals are however more likely to practice yoga (Birdee et al., 2008; Cramer et al., 2013e). Thus, yoga can be specifically considered as an alternative to other forms of physical activity for overweight/obese individuals who are not adherent to recommended physical activity regimens.

\section{Conflict of interests}

This review was supported by a grant from the Rut- and KlausBahlsen-Foundation. The funding source had no influence on the design or conduct of the review; the collection, management, analysis, or interpretation of the data; or in the preparation, review, or approval of the manuscript.

\section{Financial disclosure}

The authors declare that they have no financial disclosures.

\section{Appendix A. Complete search strategy for PubMed/Medline}

(Yoga[MeSH Terms] OR Yoga*[Title/Abstract] OR Yogic[Title/Abstract] OR Asana*[Title/Abstract] OR Pranayama[Title/Abstract]) AND (Body Weights and Measures[MeSH Terms] OR Body Weight[MeSH Terms] OR Body Composition[MeSH Terms] OR Weight*[Title/Abstract] OR Body Mass Index[Title/ Abstract] OR BMI[Title/Abstract] OR Body Fat[Title/Abstract] OR fat mass[Title/Abstract] OR Body Composition[Title/Abstract] OR Adiposity[Title/Abstract] OR Obes*[Title/Abstract] OR Overweight[Title/Abstract] OR Waist Circumference[Title/Abstract] OR Waist-Hip Ratio*[Title/Abstract]) AND (Randomized Controlled Trial [Publication Type] OR controlled clinical trial[Publication Type] OR randomized[Title/Abstract] OR placebo[Title/Abstract] OR randomly[Title/Abstract] OR trial[Title/Abstract] OR groups[Title/Abstract]) NOT (Animals[MeSH Terms] NOT humans[MeSH Terms]).

Appendix Table 1

Characteristics of the included studies. Legend: NA: not assessed, NR: not reported, f: female; m: male.

\begin{tabular}{|c|c|c|c|c|c|c|c|}
\hline Reference & Origin & $\begin{array}{l}\text { Overweight } \\
\text { as inclusion } \\
\text { criterion }\end{array}$ & Sample & Intervention & Control group & $\begin{array}{l}\text { Outcome } \\
\text { assessment } \\
\text { time point }\end{array}$ & $\begin{array}{l}\text { Outcome } \\
\text { measures: }\end{array}$ \\
\hline & & & $\begin{array}{l}\text { Sample size; mean age; gender; } \\
\text { ethnicity }\end{array}$ & $\begin{array}{l}\text { Intervention; program } \\
\text { length; frequency; } \\
\text { duration } \\
\text { Components of yoga } \\
\text { intervention }\end{array}$ & $\begin{array}{l}\text { Intervention; program length; } \\
\text { frequency; duration }\end{array}$ & & $\begin{array}{l}\text { 1. Weight } \\
\text { 2. Body mass } \\
\text { index } \\
\text { 3. Body fat } \\
\text { percentage } \\
\text { 4. Fat mass } \\
\text { (absolute) } \\
\text { 5. Waist } \\
\text { circumference } \\
\text { 6. Waist hip ratio } \\
\text { 7. Safety }\end{array}$ \\
\hline $\begin{array}{l}\text { Bera and Rajapurkar } \\
\text { (1993) }\end{array}$ & India & No & $\begin{array}{l}\text { Sample size: } \mathrm{n}=40(\mathrm{n}=20 \\
\text { each) } \\
\text { Mean age: } 12-15 \text { years } \\
\text { Gender: } 100 \% \mathrm{~m} \\
\text { Ethnicity: } \mathrm{NR}\end{array}$ & $\begin{array}{l}\text { Yoga } \\
1 \text { year } \\
3 \times / \text { week } \\
45 \text { min each }\end{array}$ & No treatment & 1 year & $\begin{array}{l}\text { 1. Weight } \\
\text { 2. NA } \\
\text { 3. Body fat } \\
\text { percentage } \\
\text { 4. Fat mass }\end{array}$ \\
\hline
\end{tabular}


Appendix Table 1 (continued)

\begin{tabular}{|c|c|c|c|c|c|c|c|}
\hline Reference & Origin & $\begin{array}{l}\text { Overweight } \\
\text { as inclusion } \\
\text { criterion }\end{array}$ & Sample & Intervention & Control group & $\begin{array}{l}\text { Outcome } \\
\text { assessment } \\
\text { time point }\end{array}$ & $\begin{array}{l}\text { Outcome } \\
\text { measures: }\end{array}$ \\
\hline & & & & $\begin{array}{l}\text { Asana } \\
\text { Pranayama } \\
\text { Meditation }\end{array}$ & & & $\begin{array}{l}\text { 5. NA } \\
\text { 6. NA } \\
\text { 7. NA }\end{array}$ \\
\hline $\begin{array}{l}\text { Blumenthal et al. } \\
\text { (1989) }\end{array}$ & USA & No & $\begin{array}{l}\text { Sample size: } \mathrm{n}=101 \text { (Yoga } \mathrm{n}= \\
34 \text {; exercise } \mathrm{n}=33 \text {, no treatment } \\
\mathrm{n}=34 \text { ) } \\
\text { Mean age: Yoga } 67.8 \pm 5.9 \text { years; } \\
\text { exercise } 66.5 \pm 4.3 \text { years; no } \\
\text { treatment } 66.8 \pm 4.3 \text { years } \\
\text { Gender: } 49.5 \% \mathrm{f} \\
\text { Ethnicity: } 96.0 \% \text { Caucasians }\end{array}$ & $\begin{array}{l}\text { Yoga } \\
16 \text { weeks } \\
2 \times / \text { week } \\
60 \text { min each } \\
\text { NR }\end{array}$ & $\begin{array}{l}\text { 1) Aerobic exercise } \\
16 \text { weeks } \\
3 \times / \text { week } \\
60 \text { min. each } \\
\text { 2) No treatment }\end{array}$ & 16 weeks & $\begin{array}{l}\text { 1. Weight } \\
\text { 2. NA } \\
\text { 3. NA } \\
\text { 4. NA } \\
\text { 5. NA } \\
\text { 6. NA } \\
\text { 7. NA }\end{array}$ \\
\hline Chen et al. (2008) & Taiwan & No & $\begin{array}{l}\text { Sample size: } \mathrm{n}=204 \text { (Yoga } \mathrm{n}= \\
64 \text {, short yoga } \mathrm{n}=59, \text { no } \\
\text { treatment } \mathrm{n}=66 \text { ) } \\
\text { Mean age: } 69.0 \pm 6.3 \text { years } \\
\text { Gender: } 72.7 \% \mathrm{f} \\
\text { Ethnicity: } \mathrm{NR}\end{array}$ & $\begin{array}{l}\text { Silver yoga } \\
24 \text { weeks } \\
3 \times / \text { week } \\
70 \text { min each } \\
\text { Asana } \\
\text { Meditation } \\
\text { Relaxation }\end{array}$ & $\begin{array}{l}\text { 1) Short yoga intervention (group } \\
\text { combined with interventional } \\
\text { group for meta-analysis) } \\
24 \text { weeks } \\
3 \times / \text { week } \\
55 \text { min. Each } \\
\text { Asana } \\
\text { Relaxation }\end{array}$ & 24 weeks & $\begin{array}{l}\text { 1. Weight } \\
\text { 2. Body mass } \\
\text { index } \\
\text { 3. NA } \\
\text { 4. NA } \\
\text { 5. NA } \\
\text { 6. NA } \\
\text { 7. NA }\end{array}$ \\
\hline Chen et al. (2010) & Taiwan & No & $\begin{array}{l}\text { Sample size: } \mathrm{n}=69 \text { (Yoga } \mathrm{n}=38 \text {, } \\
\text { control } \mathrm{n}=31) \\
\text { Mean age: } 75.4 \pm 6.7 \text { years } \\
\text { Gender: } 52.7 \% \mathrm{f} \\
\text { Ethnicity: } \mathrm{NR}\end{array}$ & $\begin{array}{l}\text { Silver Yoga } \\
24 \text { weeks } \\
3 \times / \text { week } \\
70 \text { min each } \\
\text { Asana } \\
\text { Meditation } \\
\text { Relaxation }\end{array}$ & $\begin{array}{l}\text { 2) No treatment } \\
\text { No treatment }\end{array}$ & 24 weeks & $\begin{array}{l}\text { 1. NA } \\
\text { 2. NA } \\
\text { 3. Body fat } \\
\text { percentage } \\
\text { 4. NA } \\
\text { 5. NA } \\
\text { 6. NA } \\
\text { 7. Special } \\
\text { concerns }\end{array}$ \\
\hline Cohen et al., 2008a & USA & Yes & $\begin{array}{l}\text { Sample size: } \mathrm{n}=26 \text { (Yoga } \mathrm{n}= \\
\text { 14; control } \mathrm{n}=12 \text { ) } \\
\text { Mean age: Yoga } 52 \pm 9 \text { years; } \\
\text { control } 52 \pm 8 \text { years } \\
\text { Gender: } 100 \% \mathrm{~m} \\
\text { Ethnicity: } 45 \% \text { Caucasians }\end{array}$ & $\begin{array}{l}\text { Yoga } \\
10 \text { weeks } \\
3 \text { h introductory } \\
\text { course } \\
2 \times / \text { week (weeks } 1-5 \text { ) } \\
1 \times / \text { week (weeks } \\
6-10 \text { ) } \\
90 \text { min each } \\
\text { Asanas } \\
\text { Pranayama } \\
\text { Relaxation }\end{array}$ & No intervention & 10 weeks & $\begin{array}{l}\text { 1. Weight } \\
\text { 2. Body mass } \\
\text { index } \\
\text { 3. NA } \\
\text { 4. NA } \\
\text { 5. Waist } \\
\text { circumference } \\
\text { 6. NA } \\
\text { 7. Adverse } \\
\text { events }\end{array}$ \\
\hline $\begin{array}{l}\text { Elavsky and } \\
\text { McAuley (2007a) } \\
\text { (multiple } \\
\text { publications) }\end{array}$ & USA & No & $\begin{array}{l}\text { Sample size: } \mathrm{n}=164 \text { (Yoga } \mathrm{n}=62 \\
\text { exercise } \mathrm{n}=63 \text {; usual care } \mathrm{n}=39 \text { ) } \\
\text { Mean age: Yoga } 50.0 \pm 3.7 \text { years; } \\
\text { exercise } 50.5 \pm 3.4 \text {; usual care } \\
48.6 \pm 3.5 \\
\text { Gender: } 100 \% \text { f } \\
\text { Ethnicity: } 82.6 \% \text { Caucasians; } 12.6 \% \\
\text { Blacks }\end{array}$ & $\begin{array}{l}\text { Yoga } \\
16 \text { weeks } \\
2 \times / \text { week } \\
90 \text { min each } \\
\text { Asanas } \\
\text { Mediation }\end{array}$ & $\begin{array}{l}\text { 1) Exercise } \\
16 \text { weeks } \\
2 \times / \text { week } \\
90 \text { min each } \\
\text { 2) Usual care }\end{array}$ & 16 weeks & $\begin{array}{l}\text { 1. NA } \\
\text { 2. NA } \\
\text { 3. Body fat } \\
\text { percentage (NR) } \\
\text { 4. NA } \\
\text { 5. NA } \\
\text { 6. NA } \\
\text { 7. NA }\end{array}$ \\
\hline $\begin{array}{l}\text { Harbans et al. } \\
\text { (2011) }\end{array}$ & India & Yes & $\begin{array}{l}\text { Sample size: } \mathrm{n}=63 \text { (Yoga } \mathrm{n}= \\
34 ; \text { control } \mathrm{n}=29 \text { ) } \\
\text { Age range: } 20-70 \text { years } \\
\text { Gender: NR } \\
\text { Ethnicity: NR }\end{array}$ & $\begin{array}{l}\text { Yoga } \\
8 \text { weeks } \\
2 \times / \text { day } \\
45 \text { min each }\end{array}$ & $\begin{array}{l}\text { Herbal medicine } \\
\text { Lashunadi compound }\end{array}$ & 8 weeks & $\begin{array}{l}\text { 1. Weight } \\
\text { 2. Body mass } \\
\text { index } \\
\text { 3. NA } \\
\text { 4. NA } \\
\text { 5. Waist } \\
\text { circumference } \\
\text { 6. Waist hip ratio } \\
\text { 7. Significant } \\
\text { adverse events }\end{array}$ \\
\hline Hegde et al. (2013) & India & No & $\begin{array}{l}\text { Sample size: } \mathrm{n}=29 \text { (Yoga } \mathrm{n}=14 \text {, } \\
\text { control } \mathrm{n}=15) \\
\text { Mean age: Yoga } 46.5 \pm 13.0 \text { years; } \\
\text { control } 44.7 \pm 9.6 \text { years } \\
\text { Gender: } 48.3 \% \mathrm{f} \\
\text { Ethnicity: NR }\end{array}$ & $\begin{array}{l}\text { Yoga } \\
12 \text { weeks } \\
5 \times / \text { week } \\
75-90 \text { min each } \\
\text { Asanas } \\
\text { Pranayama } \\
\text { Relaxation }\end{array}$ & No intervention & 12 weeks & $\begin{array}{l}\text { 1. NA } \\
\text { 2. Body mass } \\
\text { index } \\
\text { 3. NA } \\
\text { 4. NA } \\
\text { 5. Waist } \\
\text { circumference } \\
\text { 6. Waist hip ratio } \\
\text { 7. NA }\end{array}$ \\
\hline Kanaya et al. (2014) & USA & Yes & Sample size: $\mathrm{n}=180$ (Yoga $\mathrm{n}=$ & Restorative Yoga & Stretching & 12 months & 1. Weight \\
\hline
\end{tabular}


Appendix Table 1 (continued)

\begin{tabular}{|c|c|c|c|c|c|c|c|}
\hline Reference & Origin & $\begin{array}{l}\text { Overweight } \\
\text { as inclusion } \\
\text { criterion }\end{array}$ & Sample & Intervention & Control group & $\begin{array}{l}\text { Outcome } \\
\text { assessment } \\
\text { time point }\end{array}$ & $\begin{array}{l}\text { Outcome } \\
\text { measures: }\end{array}$ \\
\hline & & & $\begin{array}{l}\text { 91, control } n=89 \text { ) } \\
\text { Mean age: Yoga } 55 \pm 7 \text { years; } \\
\text { control } 54 \pm 7 \text { years } \\
\text { Gender: } 65.3 \% \mathrm{f} \\
\text { Ethnicity: } 62.2 \% \\
\text { Caucasians }\end{array}$ & $\begin{array}{l}48 \text { weeks } \\
2 \times / \text { week (weeks } \\
1-12 \text { ) } \\
1 \times / \text { week (weeks } \\
13-24 \text { ) } \\
1 \times / \text { month (weeks } 24 \text {, } \\
\text { weeks } 48 \text { ) } \\
\text { Asanas }\end{array}$ & $\begin{array}{l}48 \text { weeks } \\
2 \times / \text { week (weeks } 1-12 \text { ) } \\
1 \times / \text { week (weeks } 13-24 \text { ) } \\
1 \times / \text { month (weeks } 24 \text { weeks-48) }\end{array}$ & & $\begin{array}{l}\text { 2. Body mass } \\
\text { index } \\
\text { 3. Body fat } \\
\text { percentage } \\
\text { 4. NA } \\
\text { 5. Waist } \\
\text { circumference } \\
\text { 6. NA } \\
\text { 7. NA }\end{array}$ \\
\hline $\begin{array}{l}\text { Kanojia et al. } \\
\text { (2013) }\end{array}$ & India & No & $\begin{array}{l}\text { Sample size: } \mathrm{n}=50(\mathrm{n}=25 \\
\text { each) } \\
\text { Mean age: Yoga } 18.6 \pm 1.1 \text { years; } \\
\text { control } 18.1 \pm 0.8 \text { years } \\
\text { Gender: } 100 \% \mathrm{f} \\
\text { Ethnicity: } \mathrm{NR}\end{array}$ & $\begin{array}{l}\text { Yoga } \\
3 \text { months } \\
6 \times / \text { week } \\
35-40 \text { min. Each } \\
\text { Asanas } \\
\text { Pranayama } \\
\text { Meditation } \\
\text { Relaxation }\end{array}$ & No intervention & 3 months & $\begin{array}{l}\text { 1. Weight } \\
\text { 2. NA } \\
\text { 3. NA } \\
\text { 4. NA } \\
\text { 5. NA } \\
\text { 6. NA } \\
\text { 7. NA }\end{array}$ \\
\hline Khatri et al., 2007 & India & No & $\begin{array}{l}\text { Sample size: } \mathrm{n}=101 \text { (Yoga } \mathrm{n}= \\
55 \text {; control } \mathrm{n}=46 \text { ) } \\
\text { Mean age: Yoga } 54.1 \pm 8.8 \text { years; } \\
\text { control } 54.0 \pm 11.2 \text { years } \\
\text { Gender: NR } \\
\text { Ethnicity: NR }\end{array}$ & $\begin{array}{l}\text { Yoga } \\
3 \text { months }\end{array}$ & Usual care & 3 months & $\begin{array}{l}\text { 1. NA } \\
\text { 2. Body mass } \\
\text { index } \\
\text { 3. NA } \\
\text { 4. NA } \\
\text { 5. Waist } \\
\text { circumference } \\
\text { 6. NA } \\
\text { 7. NA }\end{array}$ \\
\hline Kim et al. (2013) & $\begin{array}{l}\text { South } \\
\text { Korea }\end{array}$ & Yes & $\begin{array}{l}\text { Sample size: } \mathrm{n}=41 \text { (Yoga } \mathrm{n}= \\
\text { 20; control } \mathrm{n}=21 \text { ) } \\
\text { Mean age: Yoga } 48.2 \pm 7.2 \text { years; } \\
\text { control } 50.3 \pm 8.3 \text { years } \\
\text { Gender: } 100 \% \text { f } \\
\text { Ethnicity: NR }\end{array}$ & $\begin{array}{l}\text { Hatha Yoga } \\
12 \text { weeks } \\
3 \times / \text { week } \\
60 \text { min each } \\
\text { Asanas } \\
\text { Pranayama } \\
\text { Relaxation }\end{array}$ & Usual care & 12 weeks & $\begin{array}{l}\text { 1. NA } \\
\text { 2. NA } \\
\text { 3. NA } \\
\text { 4. NA } \\
\text { 5. Waist } \\
\text { circumference } \\
\text { 6. NA } \\
\text { 7. Drop outs due } \\
\text { to side effects }\end{array}$ \\
\hline Lee et al. (2012) & Korea & Yes & $\begin{array}{l}\text { Sample size: } \mathrm{n}=16 \text { (Yoga } \mathrm{n}=8 \text {, } \\
\text { control } \mathrm{n}=8 \text { ) } \\
\text { Mean age: Yoga } 54.8 \pm 2.8 \text { years; } \\
\text { control } 54.3 \pm 2.9 \text { years } \\
\text { Gender: } 100 \% \text { f } \\
\text { Ethnicity: } \mathrm{NR}\end{array}$ & $\begin{array}{l}\text { Yoga } \\
16 \text { weeks } \\
3 \times / \text { week } \\
60 \text { min each } \\
\text { Asanas } \\
\text { Pranayama } \\
\text { Relaxation }\end{array}$ & No intervention & 16 weeks & $\begin{array}{l}\text { 1. Weight } \\
\text { 2. Body mass } \\
\text { index } \\
\text { 3. Body fat } \\
\text { percentage } \\
\text { 4. NA } \\
\text { 5. Waist } \\
\text { circumference } \\
\text { 6. NA } \\
\text { 7. NA }\end{array}$ \\
\hline $\begin{array}{l}\text { Lu and Wang } \\
\text { (2007) }\end{array}$ & China & No & $\begin{array}{l}\text { Sample size: } \mathrm{n}=63(\mathrm{n}=21 \\
\text { each) } \\
\text { Mean Age: } \mathrm{NR} \\
\text { Gender: } 100 \% \mathrm{f} \\
\text { Ethnicity: NR }\end{array}$ & $\begin{array}{l}\text { Yoga } \\
\text { Asanas } \\
\text { Relaxation }\end{array}$ & $\begin{array}{l}\text { 1) Aerobic exercise } \\
\text { 2) Aerobic + yoga (group not } \\
\text { included in meta-analysis) }\end{array}$ & 10 weeks & $\begin{array}{l}\text { 1. Weight } \\
\text { 2. Body mass } \\
\text { index } \\
\text { 3. NA } \\
\text { 4. NA } \\
\text { 5. Waist } \\
\text { circumference } \\
\text { 6. Waist hip ratio } \\
\text { 7. NA }\end{array}$ \\
\hline $\begin{array}{l}\text { Mahajan et al. } \\
\text { (1999) }\end{array}$ & India & No & $\begin{array}{l}\text { Sample size: } \mathrm{n}=53 \text { ( } \mathrm{n}=30 \text { Yoga, } \\
\mathrm{n}=23 \text { control) } \\
\text { Age range } 56-90 \text { years } \\
\text { Gender: } 100 \% \mathrm{~m} \\
\text { Ethnicity: } \mathrm{NR}\end{array}$ & $\begin{array}{l}\text { Yogic lifestyle } \\
\text { intervention } \\
4 \text { day initial } \\
\text { residential yoga camp } \\
+14 \text { weeks home } \\
\text { practice ( } 60 \text { min } \\
\text { daily) } \\
\text { Asanas } \\
\text { Pranayama } \\
\text { Meditation } \\
\text { Relaxation } \\
\text { + Vegetarian diet }\end{array}$ & $\begin{array}{l}\text { Lifestyle advice } \\
\text { Diet control and moderate } \\
\text { exercise }\end{array}$ & 14 weeks & $\begin{array}{l}\text { 1. Weight } \\
\text { 2. NA } \\
\text { 3. NA } \\
\text { 4. NA } \\
\text { 5. NA } \\
\text { 6. NA } \\
\text { 7. NA }\end{array}$ \\
\hline $\begin{array}{l}\text { Manchanda et al. } \\
\text { (2013) }\end{array}$ & India & Yes & $\begin{array}{l}\text { Sample size: } \mathrm{n}=100(\mathrm{n}=50 \\
\text { each }) \\
\text { Mean age: Yoga } 62.4 \pm 12.5 \text { years; } \\
\text { control } 56.7 \pm 12.8 \text { years } \\
\text { Gender: } 49.0 \% \mathrm{f}\end{array}$ & $\begin{array}{l}\text { Yoga } \\
1 \text { week yoga } \\
\text { instruction } \\
+12 \text { months home } \\
\text { practice }(60 \mathrm{~min}\end{array}$ & Usual care & 12 months & $\begin{array}{l}\text { 1. Weight } \\
\text { 2. Body mass } \\
\text { index } \\
\text { 3. NA } \\
\text { 4. NA }\end{array}$ \\
\hline
\end{tabular}


Appendix Table 1 (continued)

\begin{tabular}{|c|c|c|c|c|c|c|c|}
\hline Reference & Origin & $\begin{array}{l}\text { Overweight } \\
\text { as inclusion } \\
\text { criterion }\end{array}$ & Sample & Intervention & Control group & $\begin{array}{l}\text { Outcome } \\
\text { assessment } \\
\text { time point }\end{array}$ & $\begin{array}{l}\text { Outcome } \\
\text { measures: }\end{array}$ \\
\hline & & & Ethnicity: NR & $\begin{array}{l}\text { daily) } \\
\text { Asanas } \\
\text { Pranayama } \\
\text { Meditation } \\
\text { Relaxation } \\
\text { Lifestyle }\end{array}$ & & & $\begin{array}{l}\text { 5. Waist } \\
\text { circumference } \\
\text { 6. NA } \\
\text { 7. NA }\end{array}$ \\
\hline $\begin{array}{l}\text { Manjunath and } \\
\text { Telles (2012) }\end{array}$ & India & No & $\begin{array}{l}\text { Sample size: } \mathrm{n}=69(\mathrm{n}=23 \\
\text { each) } \\
\text { Mean age: Yoga } 70.1 \pm 8.3 \text { years; } \\
\text { control 1) } 72.1 \pm 9.0 \text { years; } \\
\text { control 2) } 72.3 \pm 7.4 \text { years } \\
\text { Gender: NR } \\
\text { Ethnicity: NR }\end{array}$ & $\begin{array}{l}\text { Yoga } \\
24 \text { weeks } \\
6 \times / \text { week } \\
60 \text { min each } \\
\text { Asanas } \\
\text { Pranayama } \\
\text { Meditation } \\
\text { Relaxation }\end{array}$ & $\begin{array}{l}\text { 1) Ayurvedic herbal preparation } \\
24 \text { weeks } \\
2 \times / \text { day } \\
\text { 2) No intervention }\end{array}$ & 6 months & $\begin{array}{l}\text { 1. NA } \\
\text { 2. Body mass } \\
\text { index (NR) } \\
\text { 3. NA } \\
\text { 4. NA } \\
\text { 5. NA } \\
\text { 6. NA } \\
\text { 7. NA }\end{array}$ \\
\hline $\begin{array}{l}\text { McDermott et al. } \\
\text { (2014) }\end{array}$ & India & No & $\begin{array}{l}\text { Sample size: } \mathrm{n}=41 \text { (Yoga } \mathrm{n}=21 \text {, } \\
\text { control } \mathrm{n}=20 \text { ) } \\
\text { Mean age: Yoga } 47.0 \pm 9.7 \text { years, } \\
\text { control } 47.2 \pm 9.1 \text { years } \\
\text { Gender: } 61.0 \% \mathrm{f} \\
\text { Ethnicity: NR }\end{array}$ & $\begin{array}{l}\text { Yoga } \\
8 \text { weeks } \\
3-6 \times / \text { week } \\
75 \text { min each } \\
\text { Asanas } \\
\text { Pranayama } \\
\text { Relaxation } \\
\text { Meditation }\end{array}$ & $\begin{array}{l}\text { Lifestyle advice and home-based } \\
\text { walking } \\
8 \text { weeks } \\
3-6 \times / \text { week } \\
30 \text { min each }\end{array}$ & 8 weeks & $\begin{array}{l}\text { 1. Weight } \\
\text { 2. Body mass } \\
\text { index } \\
\text { 3. NA } \\
\text { 4. NA } \\
\text { 5. Waist } \\
\text { circumference } \\
\text { 6. NA } \\
\text { 7. NA }\end{array}$ \\
\hline $\begin{array}{l}\text { Mooventhan and } \\
\text { Khode (2014) }\end{array}$ & India & No & $\begin{array}{l}\text { Sample size: } \mathrm{n}=82(\mathrm{n}=41 \\
\text { each }) \\
\text { Mean age: Yoga } 19.8 \pm 1.7 \text { years, } \\
\text { control } 19.7 \pm 2.0 \text { years } \\
\text { Gender: } 78.0 \% \mathrm{f} \\
\text { Ethnicity: NR }\end{array}$ & $\begin{array}{l}\text { Yoga } \\
2 \text { weeks } \\
6 \text { days/week } \\
10 \text { min/day } \\
\text { Pranayama }\end{array}$ & No intervention & 2 weeks & $\begin{array}{l}\text { 1. Weight } \\
\text { 2. Body Mass } \\
\text { Index } \\
\text { 3. NA } \\
\text { 4. NA } \\
\text { 5. NA } \\
\text { 6. NA } \\
\text { 7. NR }\end{array}$ \\
\hline Ray et al. (2001) & India & No & $\begin{array}{l}\text { Sample size: } \mathrm{n}=40 \text { ( } \mathrm{n}=20 \text { each }) \\
\text { Mean age: Yoga } 21.9 \pm 1.5 \text { years; } \\
\text { control } 22.7 \pm 2.0 \text { years } \\
\text { Gender: } 100 \% \mathrm{~m} \\
\text { Ethnicity: } \mathrm{NR}\end{array}$ & $\begin{array}{l}\text { Hatha Yoga } \\
6 \text { months } \\
6 \times / \text { week } \\
60 \text { min each } \\
\text { Asana } \\
\text { Pranayama } \\
\text { Relaxation } \\
\text { Meditation }\end{array}$ & $\begin{array}{l}\text { Exercise } \\
6 \text { months } \\
6 \times / \text { week } \\
60 \text { min each }\end{array}$ & 6 months & $\begin{array}{l}\text { 1. Weight } \\
\text { 2. NA } \\
\text { 3. Body fat } \\
\text { percentage } \\
\text { 4. Fat mass } \\
\text { (absolute) } \\
\text { 5. NA } \\
\text { 6. NA } \\
\text { 7. NA }\end{array}$ \\
\hline $\begin{array}{l}\text { Sakuma et al. } \\
\text { (2012) }\end{array}$ & Japan & No & $\begin{array}{l}\text { Sample size: } \mathrm{n}=123 \text { (Yoga } \mathrm{n}= \\
83 \text {, control } \mathrm{n}=40 \text { ) } \\
\text { Mean age: Yoga } 32.6 \pm 11.5 \text { years; } \\
\text { control } 35.8 \pm 13.0 \text { years } \\
\text { Gender: } 100 \% \mathrm{f} \\
\text { Ethnicity: NR }\end{array}$ & $\begin{array}{l}\text { Yogasanas } \\
2 \text { weeks } \\
7 \times / \text { week } \\
15 \text { min each } \\
\text { Asanas } \\
\text { Pranayama }\end{array}$ & No intervention & 4 weeks & $\begin{array}{l}\text { 1. Weight } \\
\text { 2. Body mass } \\
\text { index } \\
\text { 3. NA } \\
\text { 4. NA } \\
\text { 5. NA } \\
\text { 6. NA } \\
\text { 7. Adverse } \\
\text { events }\end{array}$ \\
\hline Seo et al. (2012) & Korea & Yes & $\begin{array}{l}\text { Sample size: } \mathrm{n}=20(\mathrm{n}=10 \\
\text { each) } \\
\text { Mean age: Yoga } 14.7 \pm 0.5 \text { years; } \\
\text { control: } 14.6 \pm 1.0 \text { years } \\
\text { Gender: } 100 \% \mathrm{~m} \\
\text { Ethnicity: } \mathrm{NR}\end{array}$ & $\begin{array}{l}\text { Yoga } \\
8 \text { weeks } \\
3 \times / \text { week } \\
60 \text { min each } \\
\text { Asanas } \\
\text { Relaxation }\end{array}$ & No treatment & 8 weeks & $\begin{array}{l}\text { 1. Weight } \\
\text { 2. Body mass } \\
\text { index } \\
\text { 3. Body fat } \\
\text { percentage } \\
\text { 4. Fat mass } \\
\text { (absolute) } \\
\text { 5. NA } \\
\text { 6. NA } \\
\text { 7. NA }\end{array}$ \\
\hline $\begin{array}{l}\text { Shukla and Gehlot } \\
\text { (2014) }\end{array}$ & India & Yes & $\begin{array}{l}\text { Sample size: } \mathrm{n}=60(\mathrm{n}=30 \text { each }) \\
\text { Age: } 25-55 \text { years } \\
\text { Gender: } 55.0 \% \mathrm{f} \\
\text { Ethnicity: NR }\end{array}$ & $\begin{array}{l}\text { Yoga } \\
90 \text { days Kapalabhati } \\
50 \times / \text { day; } \\
\text { Paschimottanasana } \\
20 \times / \text { day } \\
\text { Asana } \\
\text { Pranayama }\end{array}$ & $\begin{array}{l}\text { Diet } \\
90 \text { days } \\
1600 \mathrm{Kcal} / \text { day }\end{array}$ & 90 days & $\begin{array}{l}\text { 1. Weight } \\
\text { 2. Body Mass } \\
\text { Index } \\
\text { 3. Body fat } \\
\text { percentage } \\
\text { 4. Fat mass } \\
\text { (absolute, body } \\
\text { fat, visceral fat) } \\
\text { 5. Waist } \\
\text { circumference } \\
\text { 6. Waist hip ratio }\end{array}$ \\
\hline Stachenfeld et al. & USA & No & Sample size: $\mathrm{n}=17$ (Yoga $\mathrm{n}=8$ & Yoga & Aerobic exercise & 12 weeks & $\begin{array}{l}\text { 7. NA } \\
\text { 1. Weight }\end{array}$ \\
\hline
\end{tabular}


Appendix Table 1 (continued)

\begin{tabular}{|c|c|c|c|c|c|c|c|}
\hline Reference & Origin & $\begin{array}{l}\text { Overweight } \\
\text { as inclusion } \\
\text { criterion }\end{array}$ & Sample & Intervention & Control group & $\begin{array}{l}\text { Outcome } \\
\text { assessment } \\
\text { time point }\end{array}$ & $\begin{array}{l}\text { Outcome } \\
\text { measures: }\end{array}$ \\
\hline $\begin{array}{l}\text { (1998); DiPietro } \\
\text { et al. (1998) }\end{array}$ & & & $\begin{array}{l}\text { control } n=9 \text { ) } \\
\text { Mean age: Yoga } 71 \pm 2 \text { years; } \\
\text { control } 73 \pm 3 \text { years } \\
\text { Gender: } 100 \% \text { f } \\
\text { Ethnicity: NR }\end{array}$ & $\begin{array}{l}12 \text { weeks } \\
3-4 \times / \text { week } \\
60 \text { min each } \\
\text { Asanas }\end{array}$ & $\begin{array}{l}12 \text { weeks } \\
3-4 \times / \text { week } \\
40-50 \text { min each } \\
\text { Treadmill or trampoline walking }\end{array}$ & & $\begin{array}{l}\text { 2. Body mass } \\
\text { index } \\
\text { 3. NA } \\
\text { 4. NA } \\
\text { 5. NA } \\
\text { 6. NA } \\
\text { 7. NA }\end{array}$ \\
\hline Telles et al. (2013) & India & No & $\begin{array}{l}\text { Sample size: } \mathrm{n}=98 \text { (Yoga } \mathrm{n}=49 \\
\text { control } \mathrm{n}=49) \\
\text { Mean age: Yoga } 10.4 \pm 1.2 \text { years; } \\
\text { control } 10.5 \pm 1.3 \text { years } \\
\text { Gender: } 38.8 \% \\
\text { Ethnicity: NR }\end{array}$ & $\begin{array}{l}\text { Yoga } \\
3 \text { months } \\
5 \times / \text { week } \\
45 \text { min each } \\
\text { Asanas } \\
\text { Pranayama } \\
\text { Relaxation }\end{array}$ & $\begin{array}{l}\text { Physical exercise } \\
3 \text { months } \\
5 \times / \text { week } \\
45 \text { min each }\end{array}$ & 3 months & $\begin{array}{l}\text { 1. Weight (NR) } \\
\text { 2. Body mass } \\
\text { index } \\
\text { 3. NA } \\
\text { 4. Fat mass (NR) } \\
\text { 5. Waist } \\
\text { circumference } \\
\text { 6. Waist hip } \\
\text { ratio } \\
\text { 7. NA }\end{array}$ \\
\hline Telles et al. (2014) & India & Yes & $\begin{array}{l}\text { Sample size: } \mathrm{n}=68(\text { Yoga } \mathrm{n}=34 \\
\text { control } \mathrm{n}=34) \\
\text { Age: Yoga } 36.0 \pm 10.3 \text { years, } \\
\text { control } 36.8 \pm 12.1 \text { years } \\
\text { Gender: } 51.5 \% \\
\text { Ethnicity: NR }\end{array}$ & $\begin{array}{l}\text { Yoga } \\
15 \text { days } \\
2 \times / \text { day } \\
45 \text { min each } \\
\text { Asanas, Pranayama, } \\
\text { Relaxation }\end{array}$ & $\begin{array}{l}\text { Walking } \\
15 \text { days } \\
2 \times / \text { day } \\
45 \text { min each }\end{array}$ & 15 days & $\begin{array}{l}\text { 1. NA } \\
\text { 2. Body mass } \\
\text { index } \\
\text { 3. NA } \\
\text { 4. Fat mass } \\
\text { 5. Waist } \\
\text { circumference } \\
\text { 6. Waist hip } \\
\text { ratio } \\
\text { 7. Adverse } \\
\text { events }\end{array}$ \\
\hline Telles et al. (2014) & India & No & $\begin{array}{l}\text { Sample size: } n=192(\mathrm{n}=96 \\
\text { each) } \\
\text { Mean age: Yoga } 44.1 \pm 9.4 \text { years; } \\
\text { control } 42.5 \pm 9.0 \text { years } \\
\text { Gender: } 38.0 \% \mathrm{f} \\
\text { Ethnicity: NR }\end{array}$ & $\begin{array}{l}\text { Yoga } \\
12 \text { weeks } \\
3 \times / \text { week } \\
45 \text { min. Each } \\
\text { Asanas } \\
\text { Pranayama } \\
\text { Relaxation }\end{array}$ & $\begin{array}{l}\text { Lifestyle modification } \\
12 \text { weeks } \\
\text { Diet } \\
\text { Physical activity } \\
\text { Maintaining/Reducing weight }\end{array}$ & 12 weeks & $\begin{array}{l}\text { 1. Weight } \\
\text { 2. Body mass } \\
\text { index } \\
\text { 3. NA } \\
\text { 4. NA } \\
\text { 5. Waist } \\
\text { circumference } \\
\text { 6. NA } \\
\text { 7. NA }\end{array}$ \\
\hline $\begin{array}{l}\text { Tracy and Hart, } \\
2013\end{array}$ & USA & No & $\begin{array}{l}\text { Sample size: } \mathrm{n}=21 \text { (Yoga } \mathrm{n}=21 \text {, } \\
\text { control } \mathrm{n}=11) \\
\text { Mean age: } 29.0 \pm 6.1 \text { years; } \\
\text { control } 25.1 \pm 5.0 \text { years } \\
\text { Gender: } 52.4 \% \\
\text { Ethnicity: } N R\end{array}$ & $\begin{array}{l}\text { + LSM (see control) } \\
\text { Bikram Yoga } \\
8 \text { weeks } \\
3 \times / \text { week } \\
90 \text { min each. } \\
\text { Asanas }\end{array}$ & No intervention & 8 weeks & $\begin{array}{l}\text { 1. Weight (NR) } \\
\text { 2. NA } \\
\text { 3. Body fat } \\
\text { percentage } \\
\text { 4. Fat mass } \\
\text { (absolute) (NR) } \\
\text { 5. NA } \\
\text { 6. NA } \\
\text { 7. NA }\end{array}$ \\
\hline $\begin{array}{l}\text { Van Puymbroeck } \\
\text { et al. (2007) }\end{array}$ & USA & No & $\begin{array}{l}\text { Sample size: } \mathrm{n}=17 \text { (Yoga } \mathrm{n}=8 \text {, } \\
\text { control } \mathrm{n}=9 \text { ) } \\
\text { Mean age: Yoga } 55.17 \pm 15.0 \\
\text { years; control } 62.7 \pm 10.8 \text { years } \\
\text { Gender: } 69.2 \% \\
\text { Ethnicity: } 92.3 \% \text { Caucasian }\end{array}$ & $\begin{array}{l}\text { Yoga } \\
8 \text { weeks } \\
2 \times / \text { week } \\
75 \text { min each } \\
\text { Asanas } \\
\text { Pranayama }\end{array}$ & Usual care & 8 weeks & $\begin{array}{l}\text { 1. NA } \\
\text { 2. Body mass } \\
\text { index } \\
\text { 3. NA } \\
\text { 4. NA } \\
\text { 5. NA } \\
\text { 6. NA } \\
\text { 7. Adverse } \\
\text { events during } \\
\text { yoga sessions }\end{array}$ \\
\hline Yang et al. (2011) & USA & No & $\begin{array}{l}\text { Sample size: } \mathrm{n}=25 \text { (Yoga } \mathrm{n}=13 \text {, } \\
\text { control } \mathrm{n}=12 \text { ) } \\
\text { Mean age: } 51.7 \pm 4.9 \text { years } \\
\text { Gender: } 91.3 \% \mathrm{f} \\
\text { Ethnicity: } 76.0 \% \text { Caucasians }\end{array}$ & $\begin{array}{l}\text { Vinyasa Yoga } \\
12 \text { weeks } \\
2 \times / \text { week } \\
60 \text { min each } \\
\text { Asanas, Pranayama, } \\
\text { Relaxation }\end{array}$ & $\begin{array}{l}\text { General health education (written } \\
\text { information) }\end{array}$ & 12 weeks & $\begin{array}{l}\text { 1. Weight } \\
\text { 2. NA } \\
\text { 3. NA } \\
\text { 4. NA } \\
\text { 5. NA } \\
\text { 6. NA } \\
\text { 7. NA }\end{array}$ \\
\hline
\end{tabular}


Appendix Table 2

Results of the meta-analysis for studies with overweight/obese participants only.

\begin{tabular}{|c|c|c|c|c|c|c|c|c|c|c|}
\hline Outcome & Comp & Study & $\begin{array}{l}\text { Yoga } \\
\text { Mean }\end{array}$ & $\begin{array}{l}\text { Yoga } \\
\text { SD }\end{array}$ & $\mathrm{N}$ & $\begin{array}{l}\text { Control } \\
\text { Mean }\end{array}$ & $\begin{array}{l}\text { Control } \\
\text { SD }\end{array}$ & $\mathrm{N}$ & Weight & SMD [95\% CI] \\
\hline \multirow[t]{8}{*}{ Weight } & $\mathrm{UC}$ & Cohen et al. (2008a) & -0.1 & 2.7 & 12 & 2.2 & 9.0 & 12 & $19.8 \%$ & $-0.33[-1.14,0.47]$ \\
\hline & & Lee et al. (2012) & 58.06 & 4.31 & 8 & 62.88 & 4.5 & 8 & $17.8 \%$ & $-1.03[-2.10,0.03]$ \\
\hline & & Manchanda et al. (2013) & 86.91 & 12.53 & 34 & 89.14 & 13.55 & 43 & $22.3 \%$ & $-0.17[-0.62,0.28]$ \\
\hline & & Seo et al. (2012) & 74.49 & 7.06 & 10 & 78.13 & 10.44 & 10 & $19.2 \%$ & $-0.39[-1.28,0.50]$ \\
\hline & & Shukla and Gehlot (2014) & -5.2 & 1.48 & 30 & -2.16 & 1.02 & 30 & $20.9 \%$ & $-2.36[-3.03,-1.69]$ \\
\hline & & Total & & & 94 & & & 103 & $100.0 \%$ & $-0.86[-1.76,0.05]$ \\
\hline & & Overall effect & \multicolumn{8}{|c|}{$\mathrm{Z}=1.86(\mathrm{P}=0.06)$} \\
\hline & & Heterogeneity & \multicolumn{8}{|c|}{$\mathrm{Chi}^{2}=30.61, \mathrm{df}=4(\mathrm{P}<0.00001) ; \mathrm{I}^{2}=87 \%$} \\
\hline \multirow[t]{8}{*}{ BMI } & UC & Cohen et al., (2008a) & -0.1 & 0.9 & 12 & 0.8 & 2.7 & 12 & $19.6 \%$ & $-0.43[-1.24,0.38]$ \\
\hline & & Lee et al. (2012) & 24.24 & 1.43 & 8 & 26.01 & 1.61 & 8 & $16.2 \%$ & $-1.10[-2.17,-0.03]$ \\
\hline & & Manchanda et al. (2013) & 31.29 & 3.99 & 34 & 33.65 & 4.9 & 43 & $24.2 \%$ & $-0.52[-0.97,-0.06]$ \\
\hline & & Seo et al. (2012) & 27.56 & 1.5 & 10 & 29.57 & 2.97 & 10 & $18.1 \%$ & $-0.82[-1.74,0.10]$ \\
\hline & & Shukla and Gehlot (2014) & -1.97 & 0.7 & 30 & -0.76 & 0.42 & 30 & $22.0 \%$ & $-2.07[-2.70,-1.43]$ \\
\hline & & Total & & & 94 & & & 103 & $100.0 \%$ & $-0.99[-1.67,-0.31]$ \\
\hline & & Overall effect & \multicolumn{8}{|c|}{$\mathrm{Z}=2.86(\mathrm{P}=0.004)$} \\
\hline & & Heterogeneity & \multicolumn{8}{|c|}{$\mathrm{Chi}^{2}=17.10, \mathrm{df}=4(\mathrm{P}=0.002) ; \mathrm{I}^{2}=77 \%$} \\
\hline \multirow[t]{6}{*}{$\mathrm{BF}(\%)$} & UC & Lee et al. (2012) & 33.83 & 4.31 & 8 & 37.45 & 2.7 & 8 & $29.1 \%$ & $-0.95[-2.00,0.10]$ \\
\hline & & Seo et al. (2012) & 35.66 & 6.05 & 10 & 36.15 & 6.55 & 10 & $32.5 \%$ & $-0.07[-0.95,0.80]$ \\
\hline & & Shukla and Gehlot (2014) & -3.00 & 1.055 & 30 & -1.03 & 1.37 & 30 & $38.4 \%$ & $-1.59[-2.18,-1.00]$ \\
\hline & & Total & & & 48 & & & 48 & $100.0 \%$ & $-0.91[-1.86,0.04]$ \\
\hline & & Overall effect & \multicolumn{8}{|c|}{$\mathrm{Z}=1.87(\mathrm{P}=0.06)$} \\
\hline & & Heterogeneity & \multicolumn{8}{|c|}{$\mathrm{Chi}^{2}=8.02, \mathrm{df}=2(\mathrm{P}=0.02) ; \mathrm{I}^{2}=75 \%$} \\
\hline \multirow[t]{13}{*}{ WC } & UC & Cohen et al. (2008a) & -2.7 & 3.0 & 12 & -2.7 & 5.0 & 12 & $19.4 \%$ & $0.00[-0.80,0.80]$ \\
\hline & & Kim et al. 2013 & 90.0 & 5.2 & 17 & 90.52 & 9.1 & 20 & $20.7 \%$ & $-0.07[-0.71,0.58]$ \\
\hline & & Lee et al. (2012) & 85.13 & 1.36 & 8 & 86.44 & 1.95 & 8 & $17.4 \%$ & $-0.74[-1.76,0.29]$ \\
\hline & & Manchanda et al. (2013) & 95.12 & 5.12 & 34 & 98.0 & 7.3 & 43 & $22.1 \%$ & $-0.44[-0.90,0.01]$ \\
\hline & & Shukla and Gehlot (2014) & -3.53 & 1.5 & 30 & -0.96 & 0.49 & 30 & $20.6 \%$ & $-2.27[-2.93,-1.61]$ \\
\hline & & Total & & & 101 & & & 113 & $100.0 \%$ & $-0.71[-1.53,0.12]$ \\
\hline & & Overall effect & \multicolumn{8}{|c|}{$\mathrm{Z}=1.68(\mathrm{P}=0.09)$} \\
\hline & & Heterogeneity & \multicolumn{8}{|c|}{$\mathrm{Chi}^{2}=29.77, \mathrm{df}=4(\mathrm{P}<0.00001) ; \mathrm{I}^{2}=87 \%$} \\
\hline & EX & Kanaya et al. (2014) & -1.7 & 4.25 & 88 & -0.8 & 5.04 & 83 & $79.5 \%$ & $-0.19[-0.49,0.11]$ \\
\hline & & Telles et al. (2014) & 109.83 & 13.28 & 22 & 108.18 & 14.41 & 22 & $20.5 \%$ & $0.12[-0.47,0.71]$ \\
\hline & & Total & & & 110 & & & 105 & $100.0 \%$ & $-0.13[-0.40,0.14]$ \\
\hline & & Overall effect & \multicolumn{8}{|c|}{ Test for overall effect: $\mathrm{Z}=0.95(\mathrm{P}=0.34)$} \\
\hline & & Heterogeneity & \multicolumn{8}{|c|}{$\mathrm{Chi}^{2}=0.84, \mathrm{df}=1(\mathrm{P}=0.36) ; \mathrm{I}^{2}=0 \%$} \\
\hline
\end{tabular}

Appendix Table 3

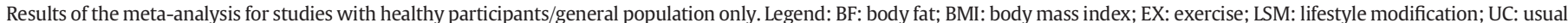
care; WC: waist circumference; WHR: waist hip Ratio.

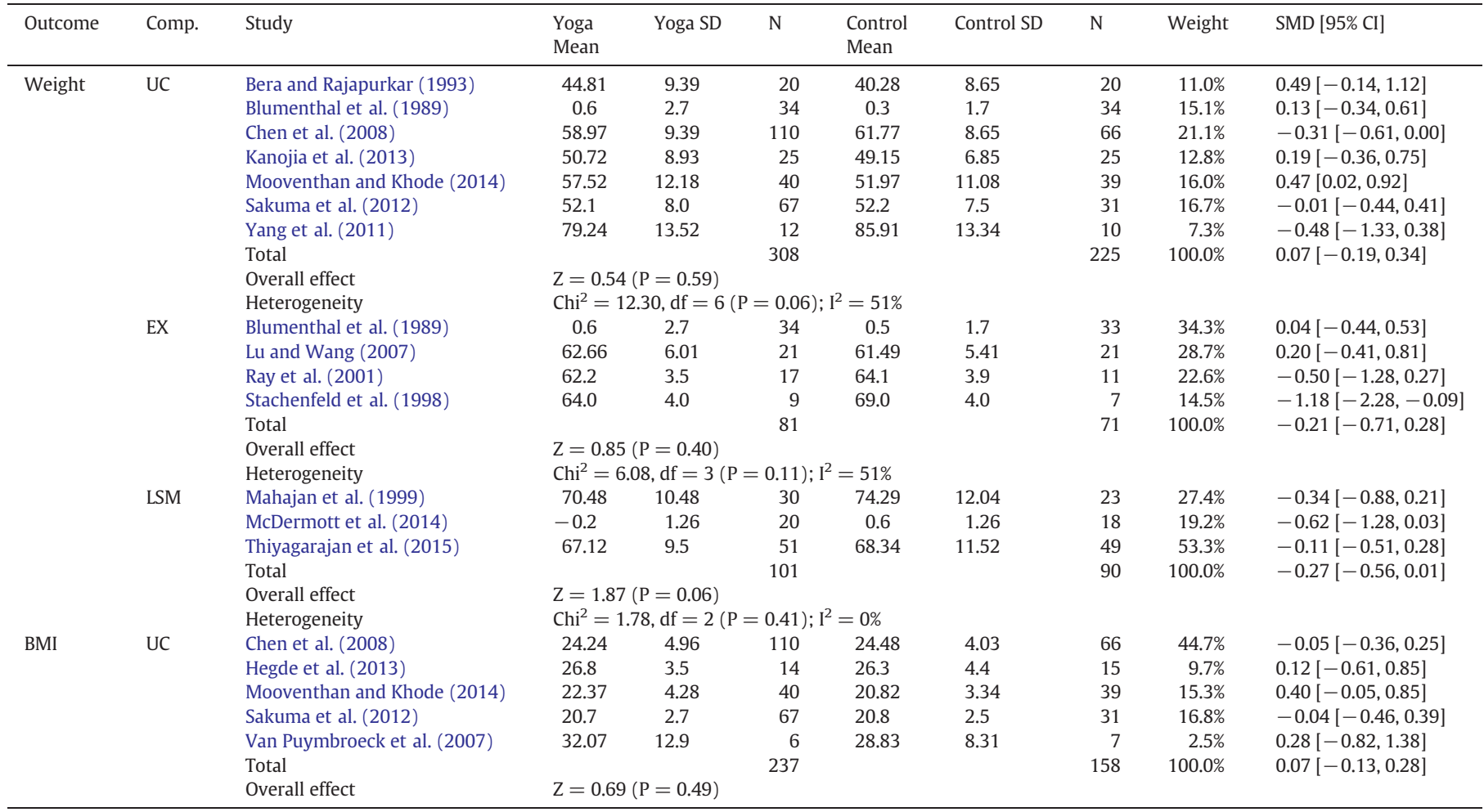


Appendix Table 3 (continued)

\begin{tabular}{|c|c|c|c|c|c|c|c|c|c|c|}
\hline Outcome & Comp. & Study & $\begin{array}{l}\text { Yoga } \\
\text { Mean }\end{array}$ & Yoga SD & $\mathrm{N}$ & $\begin{array}{l}\text { Control } \\
\text { Mean }\end{array}$ & Control SD & $\mathrm{N}$ & Weight & SMD $[95 \% \mathrm{CI}]$ \\
\hline & & Heterogeneity & $\mathrm{Chi}^{2}=$ & $\mathrm{df}=4$ & 0.54 & $=0 \%$ & & & & \\
\hline & EX & Lu and Wang (2007) & 23.08 & 1.77 & 21 & 23.4 & 1.31 & 21 & $26.9 \%$ & $-0.20[-0.81,0.40]$ \\
\hline & & Stachenfeld et al. (1998) & 27.0 & 2.7 & 9 & 26.8 & 1.7 & 7 & $10.1 \%$ & $0.08[-0.91,1.07]$ \\
\hline & & Telles et al. (2013) & 16.34 & 1.88 & 49 & 16.6 & 2.78 & 49 & $63.0 \%$ & $-0.11[-0.50,0.29]$ \\
\hline & & Total & & & 79 & & & 77 & $100.0 \%$ & $-0.11[-0.43,0.20]$ \\
\hline & & Overall effect & $\mathrm{Z}=0.7$ & $=0.48)$ & & & & & & \\
\hline & & Heterogeneity & $\mathrm{Chi}^{2}=$ & $\mathrm{df}=2(\mathrm{I}$ & $0.89)$ & $=0 \%$ & & & & \\
\hline \multirow[t]{11}{*}{$\mathrm{BF}(\%)$} & UC & Bera and Rajapurkar (1993) & 3.71 & 6.3 & 20 & 10.05 & 8.4 & 20 & $34.3 \%$ & $-0.84[-1.49,-0.19]$ \\
\hline & & Chen et al. (2010) & 28.7 & 9.27 & 31 & 29.0 & 9.03 & 24 & $36.9 \%$ & $-0.03[-0.57,0.50]$ \\
\hline & & Tracy and Hart (2013) & 27.3 & 6.3 & 10 & 21.0 & 8.4 & 11 & $28.8 \%$ & $0.81[-0.09,1.71]$ \\
\hline & & Total & & & 61 & & & 55 & $100.0 \%$ & $-0.07[-0.89,0.76]$ \\
\hline & & Overall effect & $Z=0.1$ & $=0.87)$ & & & & & & \\
\hline & & Heterogeneity & $\mathrm{Chi}^{2}=$ & $\mathrm{df}=2$ & $0.01)$ & $=77 \%$ & & & & \\
\hline & EX & Lu and Wang (2007) & 29.0 & 3.05 & 21 & 29.03 & 2.49 & 21 & $61.1 \%$ & $-0.01[-0.62,0.59]$ \\
\hline & & Ray et al. (2001) & 9.5 & 2.0 & 17 & 9.5 & 2.7 & 11 & $38.9 \%$ & $0.00[-0.76,0.76]$ \\
\hline & & Total & & & 38 & & & 32 & $100.0 \%$ & $-0.01[-0.48,0.47]$ \\
\hline & & Overall effect & $\mathrm{Z}=0.0$ & $=0.98)$ & & & & & & \\
\hline & & Heterogeneity & $\mathrm{Chi}^{2}=$ & $\mathrm{df}=1$ & $0.98)$ & $=0 \%$ & & & & \\
\hline \multirow[t]{15}{*}{ WC } & UC & Bera and Rajapurkar (1993) & 60.29 & 5.23 & 20 & 63.2 & 7.83 & 20 & $57.4 \%$ & $-0.43[-1.06,0.20]$ \\
\hline & & Hegde et al. (2013) & 89.4 & 9.1 & 14 & 90.0 & 13.7 & 15 & $42.6 \%$ & $-0.05[-0.78,0.68]$ \\
\hline & & Total & & & 34 & & & 35 & $100.0 \%$ & $-0.27[-0.74,0.21]$ \\
\hline & & Overall effect & $\mathrm{Z}=1.1$ & $=0.27)$ & & & & & & \\
\hline & & Heterogeneity & $\mathrm{Chi}^{2}=$ &, $\mathrm{df}=1$ & $0.44)$ & $=0 \%$ & & & & \\
\hline & EX & Lu and Wang (2007) & 82.05 & 0.09 & 21 & 81.02 & 3.72 & 21 & $56.3 \%$ & $0.38[-0.23,1.00]$ \\
\hline & & Stachenfeld et al. (1998) & 90.7 & 4.5 & 9 & 94.7 & 4.6 & 7 & $43.7 \%$ & $-0.83[-1.87,0.21]$ \\
\hline & & Total & & & 30 & & & 28 & $100.0 \%$ & $-0.15[-1.33,1.03]$ \\
\hline & & Overall effect & $Z=0.2$ & $=0.81)$ & & & & & & \\
\hline & & Heterogeneity & $\mathrm{Chi}^{2}=$ & , df $=1$ & $0.05)$ & $=74 \%$ & & & & \\
\hline & LSM & McDermott et al. (2014) & -4.2 & 4.71 & 20 & 0.7 & 4.71 & 18 & $42.4 \%$ & $-1.02[-1.70,-0.34]$ \\
\hline & & Thiyagarajan et al. (2015) & 82.96 & 6.26 & 51 & 85.06 & 8.0 & 49 & $57.6 \%$ & $-0.29[-0.69,0.10]$ \\
\hline & & Total & & & 71 & & & 67 & $100.0 \%$ & $-0.60[-1.30,0.11]$ \\
\hline & & Overall effect & $Z=1.6$ & $=0.10)$ & & & & & & \\
\hline & & Heterogeneity & $\mathrm{Chi}^{2}=$ & $\mathrm{df}=1$ & $0.07)$ & $=70 \%$ & & & & \\
\hline \multirow[t]{11}{*}{ WHR } & UC & Hegde et al. (2013) & 0.88 & 0.04 & 15 & 0.93 & 0.08 & 14 & $34.1 \%$ & $-0.78[-1.54,-0.02]$ \\
\hline & & Shukla and Gehlot (2014) & -0.02 & 0.01 & 30 & -0.01 & 0.01 & 30 & $65.9 \%$ & $-1.11[-1.66,-0.57]$ \\
\hline & & Total & & & 45 & & & 44 & $100.0 \%$ & $-1.00[-1.44,-0.55]$ \\
\hline & & Overall effect & $Z=4.4$ & $<0.0001)$ & & & & & & \\
\hline & & Heterogeneity & $\mathrm{Chi}^{2}=$ &, $\mathrm{df}=1$ & $0.48)$ & $=0 \%$ & & & & \\
\hline & EX & Lu and Wang (2007) & 0.69 & 0.03 & 21 & 0.79 & 0.02 & 21 & $32.6 \%$ & $-3.85[-4.90,-2.79]$ \\
\hline & & Stachenfeld et al. (1998) & 0.92 & 0.03 & 9 & 0.93 & 0.04 & 7 & $32.9 \%$ & $-0.27[-1.27,0.72]$ \\
\hline & & Telles et al. (2014) & 0.91 & 0.05 & 22 & 0.93 & 0.08 & 22 & $34.5 \%$ & $-0.29[-0.89,0.30]$ \\
\hline & & Total & & & 52 & & & 50 & $100.0 \%$ & $-1.45[-3.56,0.66]$ \\
\hline & & Overall effect & \multirow{2}{*}{\multicolumn{8}{|c|}{$\begin{array}{l}\mathrm{Z}=1.34(\mathrm{P}=0.18) \\
\mathrm{Chi}^{2}=35.44, \mathrm{df}=2(\mathrm{P}<0.00001) ; \mathrm{I}^{2}=94 \%\end{array}$}} \\
\hline & & Heterogeneity & & & & & & & & \\
\hline
\end{tabular}

Appendix Table 4

Results of the meta-analysis for studies with children/adolescent participants only. Legend: BF: body fat; UC: usual care.

\begin{tabular}{|c|c|c|c|c|c|c|c|c|c|c|}
\hline Outcome & Comp & Study & $\begin{array}{l}\text { Yoga } \\
\text { Mean }\end{array}$ & $\begin{array}{l}\text { Yoga } \\
\text { SD }\end{array}$ & $\mathrm{N}$ & $\begin{array}{l}\text { Control } \\
\text { Mean }\end{array}$ & $\begin{array}{l}\text { Control } \\
\text { SD }\end{array}$ & $\mathrm{N}$ & Weight & SMD $[95 \% \mathrm{CI}]$ \\
\hline Weight & UC & $\begin{array}{l}\text { Bera and Rajapurkar (1993) } \\
\text { Seo et al. (2012) } \\
\text { Total } \\
\text { Overall effect } \\
\text { Heterogeneity }\end{array}$ & $\begin{array}{l}44.81 \\
74.49 \\
\mathrm{Z}=0 . \\
\mathrm{Chi}^{2}=\end{array}$ & $\begin{array}{l}9.39 \\
7.06 \\
=0.81) \\
\mathrm{df}=1\end{array}$ & $\begin{array}{l}20 \\
10 \\
30\end{array}$ & $\begin{array}{l}40.28 \\
78.13 \\
=60 \%\end{array}$ & $\begin{array}{r}8.65 \\
10.44\end{array}$ & $\begin{array}{l}20 \\
10 \\
30\end{array}$ & $\begin{array}{r}56.5 \% \\
43.5 \% \\
100.0 \%\end{array}$ & $\begin{array}{l}0.49[-0.14,1.12] \\
-0.39[-1.28,0.50] \\
0.11[-0.75,0.97]\end{array}$ \\
\hline $\mathrm{BF}(\%)$ & UC & $\begin{array}{l}\text { Bera and Rajapurkar (1993) } \\
\text { Seo et al. (2012) } \\
\text { Total } \\
\text { Overall effect } \\
\text { Heterogeneity }\end{array}$ & $\begin{array}{r}3.71 \\
35.66 \\
\mathrm{Z}=1 . \\
\mathrm{Chi}^{2}=\end{array}$ & $\begin{array}{l}6.3 \\
6.05 \\
=0.17) \\
\mathrm{df}=1\end{array}$ & $\begin{array}{l}20 \\
10 \\
30\end{array}$ & $\begin{array}{l}10.05 \\
36.15 \\
=47 \%\end{array}$ & $\begin{array}{l}8.4 \\
6.55\end{array}$ & $\begin{array}{l}20 \\
10 \\
30\end{array}$ & $\begin{array}{r}57.8 \% \\
42.2 \% \\
100.0 \%\end{array}$ & $\begin{array}{l}-0.84[-1.49,-0.19] \\
-0.07[-0.95,0.80] \\
-0.52[-1.25,0.22]\end{array}$ \\
\hline
\end{tabular}

Appendix Table 5

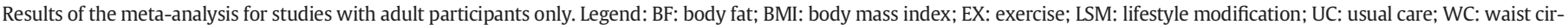
cumference; WHR: waist hip Ratio.

\begin{tabular}{|c|c|c|c|c|c|c|c|c|c|c|}
\hline Outcome & Comp. & Study & $\begin{array}{l}\text { Yoga } \\
\text { Mean }\end{array}$ & $\begin{array}{l}\text { Yoga } \\
\text { SD }\end{array}$ & $\mathrm{N}$ & $\begin{array}{l}\text { Control } \\
\text { Mean }\end{array}$ & $\begin{array}{l}\text { Control } \\
\text { SD }\end{array}$ & $\mathrm{N}$ & Weight & SMD [95\% CI] \\
\hline \multirow[t]{4}{*}{ Weight } & UC & Blumenthal et al. (1989) & 0.6 & 2.7 & 34 & 0.3 & 1.7 & 34 & $10.9 \%$ & $0.13[-0.34,0.61]$ \\
\hline & & Chen et al. (2008) & 58.97 & 9.39 & 110 & 61.77 & 8.65 & 66 & $11.9 \%$ & $-0.31[-0.61,0.00]$ \\
\hline & & Cohen et al. (2008a) & -0.1 & 2.7 & 12 & 2.2 & 9.0 & 12 & $8.6 \%$ & $-0.33[-1.14,0.47]$ \\
\hline & & Kanojia et al. (2013) & 50.72 & 8.93 & 25 & 49.15 & 6.85 & 25 & $10.4 \%$ & $0.19[-0.36,0.75]$ \\
\hline
\end{tabular}


Appendix Table 5 (continued)

\begin{tabular}{|c|c|c|c|c|c|c|c|c|c|c|}
\hline Outcome & Comp. & Study & $\begin{array}{l}\text { Yoga } \\
\text { Mean }\end{array}$ & $\begin{array}{l}\text { Yoga } \\
\text { SD }\end{array}$ & $\mathrm{N}$ & $\begin{array}{l}\text { Control } \\
\text { Mean }\end{array}$ & $\begin{array}{l}\text { Control } \\
\text { SD }\end{array}$ & $\mathrm{N}$ & Weight & SMD [95\% CI] \\
\hline & & Lee et al. (2012) & 58.06 & 4.31 & 8 & 62.88 & 4.5 & 8 & $7.0 \%$ & $-1.03[-2.10,0.03]$ \\
\hline & & Manchanda et al. (2013) & 86.91 & 12.53 & 34 & 89.14 & 13.55 & 43 & $11.0 \%$ & $-0.17[-0.62,0.28]$ \\
\hline & & Mooventhan and Khode (2014) & 57.52 & 12.18 & 40 & 51.97 & 11.08 & 39 & $11.1 \%$ & $0.47[0.02,0.92]$ \\
\hline & & Sakuma et al. (2012) & 52.1 & 8.0 & 67 & 52.2 & 7.5 & 31 & $11.2 \%$ & $-0.01[-0.44,0.41]$ \\
\hline & & Shukla and Gehlot (2014) & -5.2 & 1.48 & 30 & -2.16 & 1.02 & 30 & $9.6 \%$ & $-2.36[-3.03,-1.69]$ \\
\hline & & Yang et al. (2011) & 79.24 & 13.52 & 12 & 85.91 & 13.34 & 10 & $8.3 \%$ & $-0.48[-1.33,0.38]$ \\
\hline & & Total & & & 372 & & & 298 & $100.0 \%$ & $-0.34[-0.76,0.08]$ \\
\hline & & Overall effect & \multicolumn{8}{|c|}{$Z=1.57(P=0.12)$} \\
\hline & & Heterogeneity & \multicolumn{8}{|c|}{$\mathrm{Chi}^{2}=56.67, \mathrm{df}=9(\mathrm{P}<0.00001) ; \mathrm{I}^{2}=84 \%$} \\
\hline & EX & Blumenthal et al. (1989) & 0.6 & 2.7 & 34 & 0.5 & 1.7 & 33 & $24.4 \%$ & $0.04[-0.44,0.53]$ \\
\hline & & Kanaya et al. (2014) & -1.3 & 2.83 & 88 & -0.7 & 3.21 & 83 & $37.6 \%$ & $-0.20[-0.50,0.10]$ \\
\hline & & Lu and Wang (2007) & 62.66 & 6.01 & 21 & 61.49 & 5.41 & 21 & $18.2 \%$ & $0.20[-0.41,0.81]$ \\
\hline & & Ray et al. (2001) & 62.2 & 3.5 & 17 & 64.1 & 3.9 & 11 & $12.7 \%$ & $-0.50[-1.28,0.27]$ \\
\hline & & Stachenfeld et al. (1998) & 64.0 & 4.0 & 9 & 69.0 & 4.0 & 7 & $7.1 \%$ & $-1.18[-2.28,-0.09]$ \\
\hline & & Total & & & 169 & & & 154 & $100.0 \%$ & $-0.18[-0.49,0.14]$ \\
\hline & & Overall effect & \multicolumn{8}{|c|}{$\mathrm{Z}=1.11(\mathrm{P}=0.27)$} \\
\hline & & Heterogeneity & \multicolumn{8}{|c|}{$\mathrm{Chi}^{2}=6.21, \mathrm{df}=4(\mathrm{P}=0.18) ; \mathrm{I}^{2}=36 \%$} \\
\hline & LSM & Mahajan et al. (1999) & 70.48 & 10.48 & 30 & 74.29 & 12.04 & 23 & $27.4 \%$ & $-0.34[-0.88,0.21]$ \\
\hline & & McDermott et al. (2014) & -0.2 & 1.26 & 20 & 0.6 & 1.26 & 18 & $19.2 \%$ & $-0.62[-1.28,0.03]$ \\
\hline & & Thiyagarajan et al. (2015) & 67.12 & 9.5 & 51 & 68.34 & 11.52 & 49 & $53.3 \%$ & $-0.11[-0.51,0.28]$ \\
\hline & & Total & & & 101 & & & 90 & $100.0 \%$ & $-0.27[-0.56,0.01]$ \\
\hline & & Overall effect & \multicolumn{8}{|c|}{$Z=1.87(P=0.06)$} \\
\hline & & Heterogeneity & \multicolumn{8}{|c|}{$\mathrm{Chi}^{2}=1.78, \mathrm{df}=2(\mathrm{P}=0.41) ; \mathrm{I}^{2}=0 \%$} \\
\hline BMI & $\mathrm{UC}$ & Chen et al. (2008) & 24.24 & 4.96 & 110 & 24.48 & 4.03 & 66 & $13.7 \%$ & $-0.05[-0.36,0.25]$ \\
\hline & & Cohen et al. (2008a) & -0.1 & 0.9 & 12 & 0.8 & 2.7 & 12 & $9.4 \%$ & $-0.43[-1.24,0.38]$ \\
\hline & & Hegde et al. (2013) & 24.24 & 4.96 & 110 & 24.48 & 4.03 & 66 & $13.7 \%$ & $-0.05[-0.36,0.25]$ \\
\hline & & Lee et al. (2012) & 24.24 & 1.43 & 8 & 26.01 & 1.61 & 8 & $7.4 \%$ & $-1.10[-2.17,-0.03]$ \\
\hline & & Manchanda et al. (2013) & 31.29 & 3.99 & 34 & 33.65 & 4.9 & 43 & $12.5 \%$ & $-0.52[-0.97,-0.06]$ \\
\hline & & Mooventhan and Khode (2014) & 22.37 & 4.28 & 40 & 20.82 & 3.34 & 39 & $12.6 \%$ & $0.40[-0.05,0.85]$ \\
\hline & & Sakuma et al. (2012) & 20.7 & 2.7 & 67 & 20.8 & 2.5 & 31 & $12.7 \%$ & $-0.04[-0.46,0.39]$ \\
\hline & & Shukla and Gehlot (2014) & -1.97 & 0.7 & 30 & -0.76 & 0.42 & 30 & $10.9 \%$ & $-2.07[-2.70,-1.43]$ \\
\hline & & Van Puymbroeck et al. (2007) & 32.07 & 12.9 & 6 & 28.83 & 8.31 & 7 & $7.2 \%$ & $0.28[-0.82,1.38]$ \\
\hline & & Total & & & 417 & & & 302 & $100.0 \%$ & $-0.36[-0.77,0.05]$ \\
\hline & & Overall effect & $\mathrm{Z}=1.7$ & $=0.09)$ & & & & & & \\
\hline & & Heterogeneity & $\mathrm{Chi}^{2}=$ & $3, \mathrm{df}=8$ & $<0.00$ & $; I^{2}=83 \%$ & & & & \\
\hline & EX & Lu and Wang (2007) & 23.08 & 1.77 & 21 & 23.4 & 1.31 & 21 & $41.1 \%$ & $-0.20[-0.81,0.40]$ \\
\hline & & Stachenfeld et al. (1998) & 27.0 & 2.7 & 9 & 26.8 & 1.7 & 7 & $16.9 \%$ & $0.08[-0.91,1.07]$ \\
\hline & & Telles et al. (2014) & 36.61 & 6.54 & 22 & 33.8 & 5.76 & 22 & $42.0 \%$ & $0.45[-0.15,1.05]$ \\
\hline & & Total & & & 52 & & & 50 & $100.0 \%$ & $0.12[-0.30,0.54]$ \\
\hline & & Overall effect & $\mathrm{Z}=0.5$ & $=0.58)$ & & & & & & \\
\hline & & Heterogeneity & $\mathrm{Chi}^{2}=$ & $\mathrm{df}=2$ & $=0.33$ & $=11 \%$ & & & & \\
\hline $\mathrm{BF}(\%)$ & UC & Chen et al. (2010) & 28.7 & 9.27 & 31 & 29.0 & 9.03 & 24 & $27.0 \%$ & $-0.03[-0.57,0.50]$ \\
\hline & & Lee et al. (2012) & 33.83 & 4.31 & 8 & 37.45 & 2.7 & 8 & $22.5 \%$ & $-0.95[-2.00,0.10]$ \\
\hline & & Shukla and Gehlot (2014) & -3.0 & 1.06 & 30 & -1.03 & 1.37 & 30 & $26.6 \%$ & $-1.59[-2.18,-1.00]$ \\
\hline & & Tracy and Hart (2013) & 27.3 & 6.3 & 10 & 21.0 & 8.4 & 11 & $23.9 \%$ & $0.81[-0.09,1.71]$ \\
\hline & & Total & & & 79 & & & 73 & $100.0 \%$ & $-0.45[-1.50,0.59]$ \\
\hline & & Overall effect & $\mathrm{Z}=0.8$ & $=0.40)$ & & & & & & \\
\hline & & Heterogeneity & $\mathrm{Chi}^{2}=$ & $9, \mathrm{df}=3$ & $<0.00$ & $; I^{2}=88 \%$ & & & & \\
\hline & EX & Lu and Wang (2007) & 29.0 & 3.05 & 21 & 29.03 & 2.49 & 21 & $61.1 \%$ & $-0.01[-0.62,0.59]$ \\
\hline & & Ray et al. (2001) & 9.5 & 2.0 & 17 & 9.5 & 2.7 & 11 & $38.9 \%$ & $0.00[-0.76,0.76]$ \\
\hline & & Total & & & 38 & & & 32 & $100.0 \%$ & $-0.01[-0.48,0.47]$ \\
\hline & & Overall effect & $\mathrm{Z}=0.0$ & $=0.98)$ & & & & & & \\
\hline & & Heterogeneity & $\mathrm{Chi}^{2}=$ & $\mathrm{df}=1$ & $=0.98$ & $=0 \%$ & & & & \\
\hline WC & UC & Cohen et al. (2008a) & -2.7 & 3.0 & 12 & -2.7 & 5.0 & 12 & $16.1 \%$ & $0.00[-0.80,0.80]$ \\
\hline & & Hegde et al. (2013) & 89.4 & 9.1 & 14 & 90.0 & 13.7 & 15 & $16.6 \%$ & $-0.05[-0.78,0.68]$ \\
\hline & & Kim et al. (2013) & 90.0 & 5.2 & 17 & 90.52 & 9.1 & 20 & $17.3 \%$ & $-0.07[-0.71,0.58]$ \\
\hline & & Lee et al. (2012) & 85.13 & 1.36 & 8 & 86.44 & 1.95 & 8 & $14.2 \%$ & $-0.74[-1.76,0.29]$ \\
\hline & & Manchanda et al. (2013) & 95.12 & 5.12 & 34 & 98.0 & 7.3 & 43 & $18.6 \%$ & $-0.44[-0.90,0.01]$ \\
\hline & & Shukla and Gehlot (2014) & -3.53 & 1.5 & 30 & -0.96 & 0.49 & 30 & $17.2 \%$ & $-2.27[-2.93,-1.61]$ \\
\hline & & Total & & & 115 & & & 128 & $100.0 \%$ & $-0.60[-1.31,0.11]$ \\
\hline & & Overall effect & $Z=1.6$ & $=0.10)$ & & & & & & \\
\hline & & Heterogeneity & $\mathrm{Chi}^{2}=$ & $0, \mathrm{df}=5$ & $<0.00$ & )$; I^{2}=85 \%$ & & & & \\
\hline & EX & Kanaya et al. (2014) & -1.7 & 4.25 & 88 & -0.8 & 5.04 & 83 & $43.1 \%$ & $-0.19[-0.49,0.11]$ \\
\hline & & Lu and Wang (2007) & 82.05 & 0.09 & 21 & 81.02 & 3.72 & 21 & $22.8 \%$ & $0.38[-0.23,1.00]$ \\
\hline & & Stachenfeld et al. (1998) & 90.7 & 4.5 & 9 & 94.7 & 4.6 & 7 & $10.4 \%$ & $-0.83[-1.87,0.21]$ \\
\hline & & Telles et al. (2014) & 109.83 & 13.28 & 22 & 108.18 & 14.41 & 22 & $23.7 \%$ & $0.12[-0.47,0.71]$ \\
\hline & & Total & & & 140 & & & 133 & $100.0 \%$ & $-0.05[-0.42,0.32]$ \\
\hline & & Overall effect & $Z=0.2$ & $=0.77)$ & & & & & & \\
\hline & & Heterogeneity & $\mathrm{Chi}^{2}=$ & $\mathrm{df}=3$ & $=0.16$ & $=42 \%$ & & & & \\
\hline & LSM & McDermott et al. (2014) & -4.2 & 4.71 & 20 & 0.7 & 4.71 & 18 & $42.4 \%$ & $-1.02[-1.70,-0.34]$ \\
\hline & & Thiyagarajan et al. (2015) & 82.96 & 6.26 & 51 & 85.06 & 8.0 & 49 & $57.6 \%$ & $-0.29[-0.69,0.10]$ \\
\hline & & Total & & & 71 & & & 67 & $100.0 \%$ & $-0.60[-1.30,0.11]$ \\
\hline & & Overall effect & $\mathrm{Z}=1.6$ & $=0.10)$ & & & & & & \\
\hline & & Heterogeneity & $\mathrm{Chi}^{2}=$ & $\mathrm{df}=1$ & $=0.07$ & $=70 \%$ & & & & \\
\hline WHR & UC & Hegde et al. (2013) & 0.88 & 0.04 & 15 & 0.93 & 0.08 & 14 & $34.1 \%$ & $-0.78[-1.54,-0.02]$ \\
\hline
\end{tabular}


Appendix Table 5 (continued)

\begin{tabular}{|c|c|c|c|c|c|c|c|c|c|c|}
\hline Outcome & Comp. & Study & $\begin{array}{l}\text { Yoga } \\
\text { Mean }\end{array}$ & $\begin{array}{l}\text { Yoga } \\
\text { SD }\end{array}$ & $\mathrm{N}$ & $\begin{array}{l}\text { Control } \\
\text { Mean }\end{array}$ & $\begin{array}{l}\text { Control } \\
\text { SD }\end{array}$ & $\mathrm{N}$ & Weight & SMD $[95 \% \mathrm{CI}]$ \\
\hline & \multirow{10}{*}{ EX } & Shukla and Gehlot (2014) & -0.02 & 0.01 & 30 & -0.01 & 0.01 & 30 & $65.9 \%$ & $-1.11[-1.66,-0.57]$ \\
\hline & & Total & & & 45 & & & 44 & $100.0 \%$ & $-1.00[-1.44,-0.55]$ \\
\hline & & Overall effect & \multirow{2}{*}{\multicolumn{8}{|c|}{$\begin{array}{l}\mathrm{Z}=4.41(\mathrm{P}<0.0001) \\
\mathrm{Chi}^{2}=0.49 \mathrm{df}=1(\mathrm{P}=0.48) \cdot \mathrm{I}^{2}=0 \%\end{array}$}} \\
\hline & & Heterogeneity & & & & & \multicolumn{4}{|c|}{$\mathrm{Chi}^{2}=0.49, \mathrm{df}=1(\mathrm{P}=0.48) ; \mathrm{I}^{2}=0 \%$} \\
\hline & & Lu and Wang (2007) & 0.69 & 0.03 & 21 & 0.79 & 0.02 & 21 & $32.6 \%$ & $-3.85[-4.90,-2.79]$ \\
\hline & & Stachenfeld et al. (1998) & 0.92 & 0.03 & 9 & 0.93 & 0.04 & 7 & $32.9 \%$ & $-0.27[-1.27,0.72]$ \\
\hline & & Telles et al. (2014) & 0.91 & 0.05 & 22 & 0.93 & 0.08 & 22 & $34.5 \%$ & $-0.29[-0.89,0.30]$ \\
\hline & & Total & & & 52 & & & 50 & $100.0 \%$ & $-1.45[-3.56,0.66]$ \\
\hline & & Overall effect & \multirow{2}{*}{\multicolumn{8}{|c|}{$\begin{array}{l}\mathrm{Z}=1.34(\mathrm{P}=0.18) \\
\mathrm{Chi}^{2}=35.44, \mathrm{df}=2(\mathrm{P}<0.00001) ; \mathrm{I}^{2}=94 \%\end{array}$}} \\
\hline & & Heterogeneity & & & & & & & & \\
\hline
\end{tabular}

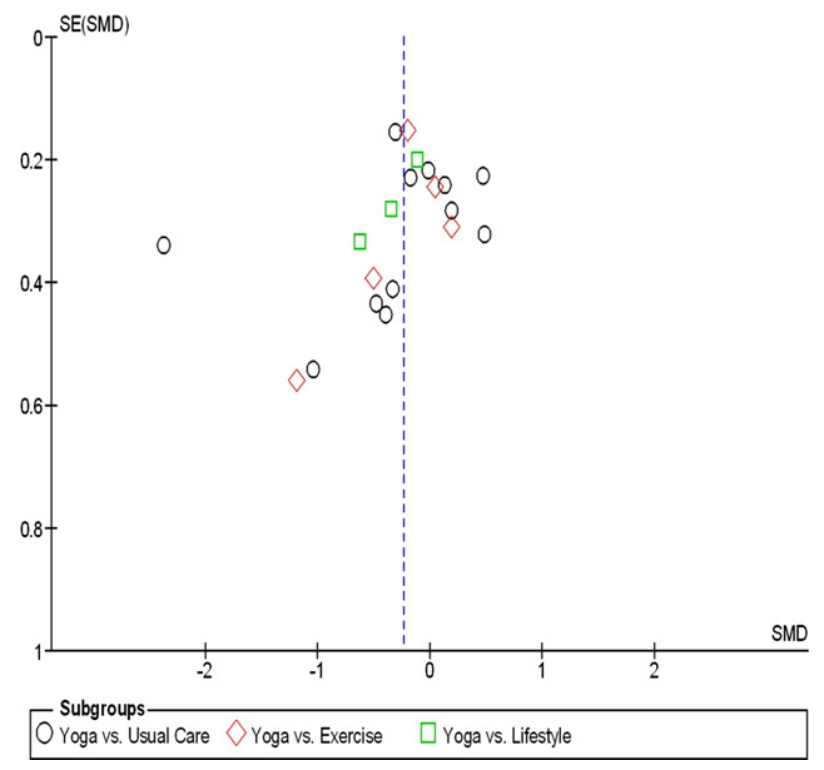

Appendix Fig. 1. Funnel plot for yoga compared to usual care, exercise and lifestyle modification for the outcome weight.

\section{Appendix B. Supplementary data}

Supplementary data to this article can be found online at http://dx. doi.org/10.1016/j.ypmed.2016.03.013.

\section{References}

Ades, P.A., Savage, P., Cress, M.E., Brochu, M., Lee, N.M., Poehlman, E.T., 2003. Resistance training on physical performance in disabled older female cardiac patients. Med. Sci. Sports Exerc. 35 (8), 1265-1270.

Ades, P.A., Savage, P.D., Brochu, M., Tischler, M.D., Lee, N.M., Poehlman, E.T., 2005. Resistance training increases total daily energy expenditure in disabled older women with coronary heart disease. J. Appl. Physiol. (1985) 98 (4), 1280-1285.

American College of Cardiology/American Heart Association Task Force on Practice Guidelines - Obesity Expert Panel 2013, 2014a. Executive summary: Guidelines (2013) for the management of overweight and obesity in adults: a report of the American College of Cardiology/American Heart Association Task Force on Practice Guidelines and the Obesity Society published by the Obesity Society and American College of Cardiology/American Heart Association Task Force on Practice Guidelines. Based on a systematic review from the The Obesity Expert Panel, 2013. Obesity 22 (Suppl. 2), S5-39.

American College of Cardiology/American Heart Association Task Force on Practice Gu idelines - Obesity Expert Panel 2013, 2014a. Expert Panel Report: Guidelines (2013) for the management of overweight and obesity in adults. Obesity 22 (Suppl. 2), S41-410.

Amita, S., Prabhakar, S., Manoj, I., Harminder, S., Pavan, T., 2009. Effect of yoga-nidra on blood glucose level in diabetic patients. Indian J. Physiol. Pharmacol. 53 (1), 97-101.

Ankad, R.B., Herur, A., Patil, S., Shashikala, G.V., Chinagudi, S., 2011. Effect of short-term pranayama and meditation on cardiovascular functions in healthy individuals. Heart Views. 12 (2), 58-62.

Ankad Roopa, B., Ankad Balachandra, S., Anita, H., Shailaja, P., Surekharani, C., Shashikala, G.V., 2011. Effect of short term pranayama and meditation on respiratory parameters in healthy individuals. Int. J. Collab. Res. Intern. Med. Public Health. 3 (6), 430-437.
Arciero, P.J., Baur, D., Connelly, S., Ormsbee, M.J., 2014. Timed-daily ingestion of whey protein and exercise training reduces visceral adipose tissue mass and improves insulin resistance: the PRISE study. J Appl Physiol (1985). 117 (6), 1-10.

Armstrong, W.J., Scott Smedley, J.M., 2003. Effects of a home-based yoga exercise program on flexibility in older women. Clinical Kinesiology. 57 (1), 1-6.

Bautista-Castano, I., Molina-Cabrillana, J., Montoya-Alonso, J.A., Serra-Majem, L., 2004. Variables predictive of adherence to diet and physical activity recommendations in the treatment of obesity and overweight, in a group of Spanish subjects. Int. J. Obes. Relat. Metab. Disord. 28 (5), 697-705.

Begum, N., Sendil Kumaran, D., Venkatesh, G., Kulkarni, S.B., 2012. Study of the utility of short course of yoga to improve reaction time. J. Clin. Diagn. Res. 6 (7 SUPPL.), 1241-1243.

Bera, T.K., Rajapurkar, M.V., 1993. Body composition, cardiovascular endurance and anaerobic power of yogic practitioner. Indian J. Physiol. Pharmacol. 37 (3), 225-228.

Bernstein, A.M., Bar, J., Ehrman, J.P., Golubic, M., Roizen, M.F., 2014. Yoga in the management of overweight and obesity. Am. J. Lifestyle Med. 8 (1), 33-41.

Bhutkar, M.V., Bhutkar, P.M., Taware, G.B., Surdi, A.D., 2011. How effective is sun salutation in improving muscle strength, general body endurance and body composition? Asian J. Sports Med. 2 (4), 259-266.

Birdee, G.S., Legedza, A.T., Saper, R.B., Bertisch, S.M., Eisenberg, D.M., Phillips, R.S., 2008. Characteristics of yoga users: results of a national survey. J. Gen. Intern. Med. 23 (10), 1653-1658.

Blumenthal, J.A., Emery, C.F., Madden, D.J., et al., 1989. Cardiovascular and behavioral effects of aerobic exercise training in healthy older men and women. J. Gerontol. 44 (5), M147-M157.

Blumenthal, J.A., Emery, C.F., Madden, D.J., et al., 1991. Effects of exercise training on cardiorespiratory function in men and women older than 60 years of age. Am. J. Cardiol. 67 (7), 633-639.

Bock, B.C., Morrow, K.M., Becker, B.M., et al., 2010. Yoga as a complementary treatment for smoking cessation: rationale, study design and participant characteristics of the Quitting-in-Balance study. BMC Complement. Altern. Med. 10, 14.

Bock, B.C., Rosen, R.K., Fava, J.L., et al., 2014. Testing the efficacy of yoga as a complementary therapy for smoking cessation: design and methods of the BreathEasy trial. Contemp. Clin. Trials. 38 (2), 321-332.

Boxer, R.S., Kleppinger, A., Brindisi, J., Feinn, R., Burleson, J.A., Kenny, A.M., 2010. Effects of dehydroepiandrosterone (DHEA) on cardiovascular risk factors in older women with frailty characteristics. Age Ageing 39 (4), 451-458.

Brady, Michele R., 2007. The effects of Hatha yoga and weight training on trait and state anxiety. Diss. Abstr. Int. 67 (8-b), 4699.

Bryan, S., Pinto Zipp, G., Parasher, R., 2012. The effects of yoga on psychosocial variables and exercise adherence: a randomized, controlled pilot study. Altern. Ther. Health Med. 18 (5), 50-59.

Bryan, S., Zipp, G.P., 2014. The effect of mindfulness meditation techniques during yoga and cycling. Altern. Complement. Ther. 20 (6), 306-316.

Bussing, A., Ostermann, T., Ludtke, R., Michalsen, A., 2012. Effects of yoga interventions on pain and pain-associated disability: a meta-analysis. J. Pain. 13 (1), 1-9.

Cadmus-Bertram, L., Littman, A.J., Ulrich, C.M., et al., 2013. Predictors of adherence to a 26week viniyoga intervention among post-treatment breast cancer survivors. J. Altern. Complement. Med. 19 (9), 751-758.

Cade, W.T., Reeds, D.N., Mondy, K.E., et al., 2010. Yoga lifestyle intervention reduces blood pressure in HIV-infected adults with cardiovascular disease risk factors. HIV Med. 11 (6), 379-388

Čajka, V., Sovová, E., Pastucha, D., et al., 2012. Risk factors for cardiovascular diseases in regular yoga practitioners in comparison with general population. Prakticky Lekar. $92(1), 41-44$.

Cajka, V., Sovová, E., Pastucha, D., et al., 2013. Life style analysis of yoga exercisers in comparison with the general population Introduction. Prakticky Lekar. 93 (6), $260-263$.

Carei, T.R., Fyfe-Johnson, A.L., Breuner, C.C., Brown, M.A., 2010. Randomized controlled clinical trial of yoga in the treatment of eating disorders. J. Adolesc. Health. 46 (4), 346-351.

Castellani, W., Ianni, L., Ricca, V., Mannucci, E., Rotella, C.M., 2003. Adherence to structured physical exercise in overweight and obese subjects: a review of psychological models. Eat. Weight Disord. 8 (1), 1-11.

Ce, E., Maggioni, M.A., Boniello, S., Veicsteinas, A., Merati, G., 2015. Anthropometric and physiologic profiles of female professional yoga practitioners and energy expenditure during asanas execution. J. Sports Med. Phys. Fitness. 55 (1-2), 51-57. 
Cecchini, M., Sassi, F., Lauer, J.A., Lee, Y.Y., Guajardo-Barron, V., Chisholm, D., 2010 Tackling of unhealthy diets, physical inactivity, and obesity: health effects and costeffectiveness. Lancet 376 (9754), 1775-1784.

Chatterjee, S., Mondal, S., 2014. Effect of regular yogic training on growth hormone and dehydroepiandrosterone sulfate as an endocrine marker of aging. Evid. Based Complement. Alternat. Med. 2014, 240581.

Chaya, M.S., Kurpad, A.V., Nagendra, H.R., Nagarathna, R., 2006. The effect of long term combined yoga practice on the basal metabolic rate of healthy adults. BMC Complement. Altern. Med. 6, 28.

Chaya, M.S., Ramakrishnan, G., Shastry, S., et al., 2008. Insulin sensitivity and cardiac autonomic function in young male practitioners of yoga. Natl Med. J. India 21 (5), 217-221.

Cheema, B.S., Marshall, P.W., Chang, D., Colagiuri, B., MacHliss, B., 2011. Effect of an office worksite-based yoga program on heart rate variability: a randomized controlled trial. BMC Public Health 11.

Chen, K.M., Chen, M.H., Hong, S.M., Chao, H.C., Lin, H.S., Li, C.H., 2008. Physical fitness of older adults in senior activity centres after 24 -week silver yoga exercises. J. Clin. Nurs. 17 (19), 2634-2646.

Chen, K.M., Fan, J.T., Wang, H.H., Wu, S.J., Li, C.H., Lin, H.S., 2010. Silver yoga exercises improved physical fitness of transitional frail elders. Nurs. Res. 59 (5), 364-370.

Cheung, C., Wyman, J., Resnick, B., 2012. Is yoga effective for knee osteoarthritis in older women? Osteoarthritis cart. 20, S280.

Cheung, C., Wyman, J.F., Resnick, B., Savik, K., 2014. Yoga for managing knee osteoarthritis in older women: a pilot randomized controlled trial. BMC Complement. Altern. Med. $14,160$.

Chong, C.S., Tsunaka, M., Tsang, H.W., Chan, E.P., Cheung, W.M., 2011. Effects of yoga on stress management in healthy adults: a systematic review. Altern. Ther. Health Med. 17 (1), 32-38.

Choudhary, A., Mishra, J., 2013. Effect of 16 weeks yogic intervention in premenstrual syndrome. Int. J. Pharm. Bio Sciences. 4 (1), B207-B212.

Chu, P., Gotink, R.A., Yeh, G.Y., Goldie, S.J., Hunink, M.M., 2014a. The effectiveness of yoga in modifying risk factors for cardiovascular disease and metabolic syndrome: a systematic review and meta-analysis of randomized controlled trials. Eur. J. Prev. Cardiol.

Chu, P., Gotink, R.A., Yeh, G.Y., Goldie, S.J., Hunink, M.M., 2014b. The effectiveness of yoga in modifying risk factors for cardiovascular disease and metabolic syndrome: a systematic review and meta-analysis of randomized controlled trials. Eur. J. Prev. Cardiol. Epub ahead of print. doi: 10.1177/2047487314562741.

Clarke, T.C., Black, L.I., Stussman, B.J., Barnes, P.M., RL, Nahin, 2002. Trends in the use of complementary health approaches among adults: United States. Natl, Health Stat. Report. 2015 (79), 1-16.

Cohen, J., 1988. Statistical Power Analysis for the Behavioral Sciences. Lawrence Erlbaum Associates, Hillsdale.

Cohen, B.E., Chang, A.A., Grady, D., Kanaya, A.M., 2008a. Restorative yoga in adults with metabolic syndrome: a randomized, controlled pilot trial. Metab. Syndr. Relat. Disord. 6 (3), 223-229.

Cohen, B.E., Chang, A.A., Grady, D., Kanaya, A.M., 2008b. Restorative yoga in adults with metabolic syndrome: a randomized, controlled pilot trial. Metab. Syndr. Relat. Disord. 6 (3), 223-229.

Cohen, D.L., Bowler, A., Fisher, S.A., et al., 2013. Lifestyle modification in blood pressure study II (LIMBS): study protocol of a randomized controlled trial assessing the efficacy of a 24 week structured yoga program versus lifestyle modification on blood pressure reduction. Contemp. Clin. Trials. 36 (1), 32-40.

Corey, S., Epel, E., Schembri, M., et al., 2014. Effect of restorative yoga vs. stretching on salivary cortisol and psychosocial outcomes in individuals with the metabolic syndrome: the PRYSMS randomized controlled trial. J. Altern. Complement. Med. 20 (5), A22.

Cramer, H., Lauche, R., Haller, H., Dobos, G., 2013a. A systematic review and meta-analysis of yoga for low back pain. Clin. J. Pain. 29 (5), 450-460.

Cramer, H., Lauche, R., Langhorst, J., Dobos, G., 2013b. Yoga for rheumatic diseases: a systematic review. Rheumatology (Oxford) 52 (11), 2025-2030.

Cramer, H., Lauche, R., Langhorst, J., Dobos, G., 2013c. Yoga for depression: a systematic review and meta-analysis. Depress Anxiety. 30 (11), 1068-1083.

Cramer, H., Krucoff, C., Dobos, G., 2013d. Adverse events associated with yoga: a systematic review of published case reports and case series. PLoS ONE 8 (10), e75515.

Cramer, H., Lauche, R., Langhorst, J., Paul, A., Michalsen, A., Dobos, G., 2013e. Predictors of yoga use among internal medicine patients. BMC Complement. Altern. Med. 13, 172.

Cramer, H., Lauche, R., Dobos, G., 2014a. Characteristics of randomized controlled trials of yoga: a bibliometric analysis. BMC Complement. Altern. Med. 14, 328.

Cramer, H., Lauche, R., Haller, H., Steckhan, N., Michalsen, A., Dobos, G., 2014b. Effects of yoga on cardiovascular disease risk factors: a systematic review and meta-analysis. Int. J. Cardiol. 173 (2), 170-183.

Cramer, H., Lauche, R., Ward, L., Steel, A., Dobos, G., Zhang, Y., 2015a. Prevalence, pattern and predictors of yoga use in the US: results of a representative survey. Am. J. Prev. Med.

Cramer, H., Ward, L., Saper, R., Fishbein, D., Dobos, G., Lauche, R., 2015b. The safety of yoga: a systematic review and meta-analysis of randomized controlled trials. Am J Epidemiol. Epub ahead of print. doi: 10.1093/aje/kwv071.

Dallman, M.F., Pecoraro, N., Akana, S.F., et al., 2003. Chronic stress and obesity: a new view of "comfort food". Proc. Natl. Acad. Sci. U. S. A. 100 (20), 11696-11701.

Dandekar, P.D., 2013. Impact of short term training of anulom vilom pranayam on blood pressure and pulse rate in healthy volunteers. Int. J. Res. Ayurveda Pharm. 4 (2), 253-256.

DeBar, L.L., Stevens, V.J., Perrin, N., et al., 2012. A primary care-based, multicomponent lifestyle intervention for overweight adolescent females. Pediatrics 129 (3), e611-e620.
Dhananjai, S., Sadashiv, Tiwari, S., Dutt, K., Kumar, R., 2013. Reducing psychological distress and obesity through Yoga practice. Int. J. Yoga. 6 (1), 66-70.

De Michelis, E., 2005. A History of Modern Yoga: Patanjali and Western Esotericism. Continuum International Publishing Group, London, UK.

DiPietro, L., Seeman, T.E., Stachenfeld, N.S., Katz, L.D., Nadel, E.R., 1998. Moderate-intensity aerobic training improves glucose tolerance in aging independent of abdominal adiposity. J. Am. Geriatr. Soc. 46 (7), 875-879.

Ebnezar, J., Nagarathna, R., Yogitha, B., Nagendra, H.R., 2012. Effects of an integrated approach of hatha yoga therapy on functional disability, pain, and flexibility in osteoarthritis of the knee joint: a randomized controlled study. J. Altern. Complement. Med. 18 (5), 463-472.

Egger, M., Davey Smith, G., Schneider, M., Minder, C., 1997. Bias in meta-analysis detected by a simple, graphical test. BMJ 315 (7109), 629-634.

Elavsky, S., McAuley, E., 2007a. Exercise and self-esteem in menopausal women: a randomized controlled trial involving walking and yoga. Am. J. Health Promot. 22 (2), 83-92.

Elavsky, S., McAuley, E., 2007b. Physical activity and mental health outcomes during menopause: a randomized controlled trial. Ann. Behav. Med. 33 (2), 132-142.

Elavsky, S., McAuley, E., 2007c. Lack of perceived sleep improvement after 4-month structured exercise programs. Menopause 14 (3 Pt 1), 535-540.

Emery, C.F., Blumenthal, J.A., 1990. Perceived change among participants in an exercise program for older adults. Gerontologist 30 (4), 516-521.

Flaherty, M., 2014. Influence of yoga on body image satisfaction in men. Percept. Mot. Skills 119 (1), 203-214.

Feuerstein, G., 1998. The Yoga Tradition. Hohm Press, Prescott.

Goncalves, L.C., Vale, R.G., Barata, N.J., Varejao, R.V., Dantas, E.H., 2011. Flexibility, functional autonomy and quality of life (QoL) in elderly yoga practitioners. Arch. Gerontol. Geriatr. 53 (2), 158-162.

Gordon, L., Morrison, E.Y., McGrowder, D.A., et al., 2008. Changes in clinical and metabolic parameters after exercise therapy in patents with type 2 diabetes. Arch. Med. Sci. 4 (4), 427-437.

Göring, A., Möllenbeck, D., Schwarz, G., 2013. The influence of dynamic yoga styles on spinal flexibility. Deutsche Zeitschrift fur Sportmedizin. 64 (9), 280-283.

Guarracino, J.L., Savino, S., Edelstein, S., 2006. Yoga participation is beneficial to obesity prevention, hypertension control, and positive quality of life. Top. Clin. Nutr. 21 (2), $108-113$.

Haaz, S., Bartlett, S.J., 2011. Yoga for arthritis: a scoping review. Rheum. Dis. Clin. N. Am. 37 (1), 33-46

Halder, K., Chatterjee, A., Pal, R., Tomer, O.S., Saha, M., 2015. Age related differences of selected Hatha yoga practices on anthropometric characteristics, muscular strength and flexibility of healthy individuals. Int. J. Yoga. 8 (1), 37-46.

Harbans, S., Anjali, S., Smita, J., 2011. Comparative clinical evaluation of the antidyslipidaemic effects of lashunadi compound and yogic exercises in patients of metabolic syndrome. Ind. J. Trad. Knowl. 10 (4), 651-656.

Hagins, M., Moore, W., Rundle, A., 2007. Does practicing hatha yoga satisfy recommendations for intensity of physical activity which improves and maintains health and cardiovascular fitness? BMC Complement Altern Med. 7, 40.

Hartley, L., Dyakova, M., Holmes, J., et al., 2014. Yoga for the primary prevention of cardiovascular disease. Cochrane Database Syst. Rev. 5, CD010072.

Hegde, S.V., Adhikari, P., Kotian, S., Pinto, V.J., D'Souza, S., D'Souza, V., 2011. Effect of 3 month yoga on oxidative stress in type 2 diabetes with or without complications: a controlled clinical trial. Diabetes Care 34 (10), 2208-2210.

Hegde, S.V., Adhikari, P., Shetty, S., Manjrekar, P., D'Souza, V., 2013. Effect of community-based yoga intervention on oxidative stress and glycemic parameters in prediabetes: a randomized controlled trial. Complement. Ther. Med. 21 (6), 571-576.

Herur, A., Kolagi, S., Chinagudi, S., 2011. Effect of body mass index and gender on the cardiovascular and mental response to yoga. Biomed. Res. 22 (4), 499-505.

Higgins, J.P., Thompson, S.G., Deeks, J.J., Altman, D.G., 2003. Measuring inconsistency in meta-analyses. BMJ 327 (7414), 557-560.

Higgins, J.P.T., Green, S., 2008. Cochrane Handbook for Systematic Reviews of Interventions. West Sussex, John Wiley \& Sons Ltd.

Hoogbruin, A.L., Innes, K.E., Dong, R., Garg, A.K., 2012. Assessing the effects of yoga on cardiovascular disease (CVD) risk in postmenopausal South Asian women: methodological challenges. Circulation 125 (19), e833-e834.

Hunter, S.D., Dhindsa, M.S., Cunningham, E., et al., 2013. The effect of Bikram yoga on arterial stiffness in young and older adults. J. Altern. Complement. Med. 19 (12), 930-934.

Ikai, S., Uchida, H., Suzuki, T., Tsunoda, K., Mimura, M., Fujii, Y., 2013. Effects of yoga therapy on postural stability in patients with schizophrenia-spectrum disorders: a single-blind randomized controlled trial. J. Psychiatr. Res. 47 (11), 1744-1750.

Innes, K.E., Vincent, H.K., 2007. The influence of yoga-based programs on risk profiles in adults with type 2 diabetes mellitus: a systematic review. Evid. Based Complement. Alternat. Med. 4 (4), 469-486.

Innes, K.E., Selfe, T.K., 2012. The effects of a gentle yoga program on sleep, mood, and blood pressure in older women with restless legs syndrome (RLS): a preliminary randomized controlled trial. Evid. Based Complement. Alternat. Med. 2012, 294058.

Iyengar, B.K.S., 1966. Light on Yoga. Schocken Books, New York.

Jayasinghe, S.R., 2004. Yoga in cardiac health (a review). Eur. J. Cardiovasc. Prev. Rehabil. 11 (5), 369-375.

Jorrakate, C., Kongsuk, J., Pongduang, C., Sadsee, B., Chanthorn, P., 2015. Effect of yoga training on one leg standing and functional reach tests in obese individuals with poor postural control. J. Phys. Ther. Sci. 27 (1), 59-62.

Kanaya, A.M., Araneta, M.R.G., Pawlowsky, S.B., et al., 2014. Restorative yoga and metabolic risk factors: the practicing restorative yoga vs. stretching for the metabolic syndrome (PRYSMS) randomized trial. J. Diabetes Compl. 28 (3), 406-412. 
Kandula NR, Patel Y, Dave S, et al. The South Asian Heart Lifestyle Intervention (SAHELI) study to improve cardiovascular risk factors in a community setting: design and methods. Contemp. Clin. Trials. 2013;36(2):479-87. http://onlinelibrary.wiley.com/ o/cochrane/clcentral/articles/799/CN-00913799/frame.html. http://ac.els-cdn.com/ S1551714413001584/1-s2.0-S1551714413001584-main.pdf? tid=940b436e-ca2b11e4-913f-00000aacb35e\&acdnat $=1426325104$ 865b7af0f1e0a9977157584fb0251573.

Kanojia, S., Sharma, V.K., Gandhi, A., Kapoor, R., Kukreja, A., Subramanian, S.K., 2013. Effect of yoga on autonomic functions and psychological status during both phases of menstrual cycle in young healthy females. J. Clin. Diagn. Res. 7 (10), 2133-2139.

Kenny, A.M., Boxer, R.S., Kleppinger, A., Brindisi, J., Feinn, R., Burleson, J.A., 2010. Dehydroepiandrosterone (DHEA) improves muscle strength and physical function but not bone mineral density in frail older women. J. Am. Geriatr. Soc. 58, S98.

Khatri, D., Mathur, K.C., Gahlot, S., Jain, S., Agrawal, R.P., 2007. Effects of yoga and meditation on clinical and biochemical parameters of metabolic syndrome. Diabetes Res. Clin. Pract. 78 (3), e9-10.

Kim, E., Park, S., Kwon, Y., 2008. The effects of combined exercise on functional fitness and risk factors of metabolic syndrome in the older women. Jap. J. Phys. Fitness Sports Med. 57 (2), 207-215.

Kim, H.N., Ryu, J., Kim, K.S., Song, S.W., 2013. Effects of yoga on sexual function in women with metabolic syndrome: a randomized controlled trial. J. Sex. Med. 10 (11), 2741-2751.

Kim, S.S., Min, W.K., Kim, J.H., Lee, B.H., 2014. The effects of vr-based wii fit yoga on physical function in middle-aged female LBP patients. J. Phys. Ther. Sci. 26 (4), 549-552.

Krejci, M., 2011. Yoga training application in overweight control of seniors with arthritis/ osteoarthritis. Fizjoterapia. 19 (2), 3-8.

Kristal, A.R., Littman, A.J., Benitez, D., White, E., 2005. Yoga practice is associated with attenuated weight gain in healthy, middle-aged men and women. Altern. Ther. Health Med. 11 (4), 28-33.

Kubo, A., Hung, Y.Y., Ritterman, J., 2011. Yoga for heart failure patients: a feasibility pilot study with a multiethnic population. Int. J. Yoga Therap. 21, 77-83.

LaCroix, A.Z., Sternfeld, B., Caan, B., et al., 2012. Results from the MsFLASH randomized controlled trial of yoga, aerobic exercise \& omega-3 supplementation for relief of vasomotor symptoms. Menopause 19 (12), 1369.

Lai, X.J., Xu, F.P., Wan, P., 2010. Comparison of the sensible perspiration \& the change of body fat after dynamic exercise \& complex static exercise. 4th Internationa Conference on Bioinformatics and Biomedical Engineering.

Lee, J.A., Kim, J.W., Kim, D.Y., 2012. Effects of yoga exercise on serum adiponectin and metabolic syndrome factors in obese postmenopausal women. Menopause 19 (3) 296-301.

Littman, A.J., Bertram, L.C., Ceballos, R., et al., 2012. Randomized controlled pilot trial of yoga in overweight and obese breast cancer survivors: effects on quality of life and anthropometric measures. Support. Care Cancer 20 (2), 267-277.

Liu, X.C., Pan, L., Hu, Q., Dong, W.P., Yan, J.H., Dong, L., 2014. Effects of yoga training in patients with chronic obstructive pulmonary disease: a systematic review and meta-analysis. J. Thorac. Dis. 6 (6), 795-802.

Lu, C., Wang, B., 2007. A study on effects of aerobics combined with yoga exercise on physical training. Chin. J. Rehabil. Med. 22 (10), 885-887.

Macy, D., 2008. Yoga journal releases 2008 "Yoga in America" market study. http:// www.yogajournal.com/advertise/press_releases/10. Yoga J.

Madanmohan, Mahadevan S.K., Balakrishnan, S., Gopalakrishnan, M., ES, Prakash, 2008. Effect of six weeks yoga training on weight loss following step test, respiratory pressures, handgrip strength and handgrip endurance in young healthy subjects. Indian J. Physiol. Pharmacol. 52 (2), 164-170.

Mahajan, A.S., Reddy, K.S., Sachdeva, U., 1999. Lipid profile of coronary risk subjects following yogic lifestyle intervention. Indian Heart J. 51 (1), 37-40.

Malhotra, V., Singh, S., Tandon, O.P., Sharma, S.B., 2005. The beneficial effect of yoga in diabetes. Nepal Med. Coll. J. 7 (2), 145-147.

Malhotra, V., Singh, S., Sharma, S.B., et al., 2010. The status of NIDDM patients after yoga asanas: assessment of important parameters. J Clin Diagn Res. 4 (3), 2652-2667.

Manchanda, S.C., Narang, R., Reddy, K.S., et al., 2000. Retardation of coronary atherosclerosis with yoga lifestyle intervention. J. Assoc. Phys. India. 48 (7), 687-694.

Manchanda, S.C., Mehotra, U.C., Makhija, A., Mohanty, A., Dhawan, S., Sawhney, J.P.S., 2013. Reversal of early atherosclerosis in metabolic syndrome by yoga - a randomized controlled trial. J Yoga Phys Ther. 3 (1), 132

Manchanda, S.C., Madan, K., 2014. Yoga and meditation in cardiovascular disease. Clin. Res. Cardiol. 103 (9), 675-680.

Maninder, B., Seema, D., Shema, N., 2013. Influence of pranayamas and yoga-asanas on blood glucose,lipid profile and hba1c in type 2 diabetes. Int. J. Pharm. Biosci. 4 (1), B169-B172.

Manjunath, N., Telles, S., 2012. Yoga for healthy aging. Sense. 2 (2), 32-41.

McCaffrey, R., Ruknui, P., Hatthakit, U., Kasetsomboon, P., 2005. The effects of yoga on hypertensive persons in Thailand. Holist. Nurs. Pract. 19 (4), 173-180

McDermott, K.A., Rao, M.R., Nagarathna, R., et al., 2014. A yoga intervention for type 2 diabetes risk reduction: a pilot randomized controlled trial. BMC Complement Altern. Med. 14, 212.

McIver, S., O'Halloran, P., McGartland, M., 2009. Yoga as a treatment for binge eating disorder: a preliminary study. Complement. Ther. Med. 17 (4), 196-202.

Mclver, S., 2010. Yoga may help manage binge eating disorder. Focus. Altern. Complement. Ther. 15 (1), 43-44

Miles, S.C., Chun-Chung, C., Hsin-Fu, L., et al., 2013. Arterial blood pressure and cardiovascular responses to yoga practice. Altern. Ther. Health Med. 19 (1), 38-45.

Mitchell, K.S., Mazzeo, S.E., Rausch, S.M., Cooke, K.L., 2007. Innovative interventions for disordered eating: evaluating dissonance-based and yoga interventions. Int. J. Eat Disord. 40 (2), 120-128.
Mody, B.S., 2011. Acute effects of Surya Namaskar on the cardiovascular \& metabolic system. J. Bodyw. Mov. Ther. 15 (3), 343-347.

Moher, D., Liberati, A., Tetzlaff, J., Altman, D.G., 2009. Preferred reporting items for systematic reviews and meta-analyses: the PRISMA statement. BMJ 339, b2535.

Mooventhan, A., Khode, V., 2014. Effect of Bhramari pranayama and OM chanting on pulmonary function in healthy individuals: a prospective randomized control trial. Int. J. Yoga. 7 (2), 104-110.

Murthy, S.N., Rao, N.S.N., Nandkumar, B., Kadam, A., 2011. Role of naturopathy and yoga treatment in the management of hypertension. Complement. Ther. Clin. Pract. 17 (1), 9-12.

Murugesan, R., Govindarajulu, N., Bera, T.K., 2000. Effect of selected yogic practices on the management of hypertension. Indian J. Physiol. Pharmacol. 44 (2), 207-210.

Nagarathna, R., Nagendra, H.R., 1987. Intergrated approach of yoga theapy in the management of diabetes mellitus. IEEE/Eng Med. Biol. Soc. Ann. Conf. 1593-1594.

Narendran, S., Nagarathna, R., Gunasheela, S., Nagendra, H.R., 2005a. Efficacy of yoga in pregnant women with abnormal Doppler study of umbilical and uterine arteries. J. Indian Med. Assoc. 103 (1), 12-14 16-7.

Narendran, S., Nagarathna, R., Narendran, V., Gunasheela, S., Nagendra, H.R., 2005b. Efficacy of yoga on pregnancy outcome. J. Altern. Complement. Med. 11 (2), 237-244.

National Health and Medical Research Council, 2013. Clinical Practice Guidelines for the Management of Overweight and Obesity in Adults, Adolescents and Children in Australia. National Health and Medical Research Council, Melbourne.

National Center for Health Statistics, 2015. Health, United States, 2014: With Special Feature on Adults Aged 55-64. National Center for Health Statistic, Hyattsville, MD.

Neumark-Sztainer, D., Eisenberg, M.E., Wall, M., Loth, K.A., 2011. Yoga and pilates: associations with body image and disordered-eating behaviors in a population-based sample of young adults. Int. J. Eat Disord. 44 (3), 276-280.

Nidhi, R., Padmalatha, V., Nagarathna, R., Ram, A., 2012. Effect of a yoga program on glucose metabolism and blood lipid levels in adolescent girls with polycystic ovary syndrome. Int. J. Gynaecol. Obstet. 118 (1), 37-41.

Nidhi, R., Padmalatha, V., Nagarathna, R., Amritanshu, R., 2013a. Effects of a holistic yoga program on endocrine parameters in adolescents with polycystic ovarian syndrome: a randomized controlled trial. J. Altern. Complement. Med. 19 (2), 153-160.

Nidhi, R., Padmalatha, V., Nagarathna, R., Amritanshu, R., 2013b. Effect of yoga program on quality of life in adolescent polycystic ovarian syndrome: a randomized control trial. Appl. Res. Qual. Life. 8 (3), 373-383.

Nishanth, S., Madanmohan, T., Das, A.K., Ramkumar, T., Senthilkumar, S., 2011. Effect of 12 week yoga therapy as a lifestyle intervention in patients of type 2 diabetes mellitus with distal symmetric polyneuropathy. Indian J. Physiol. Pharmacol. 55 (5 suppl. 1), 64

Pal, A., Srivastava, N., Tiwari, S., et al., 2011. Effect of yogic practices on lipid profile and body fat composition in patients of coronary artery disease. Complement Ther. Med. 19 (3), 122-127.

Pal, A., Srivastava, N., Narain, V.S., Agrawal, G.G., Rani, M., 2013. Effect of yogic intervention on the autonomic nervous system in the patients with coronary artery disease: a randomized controlled trial. East Mediterr. Health J. 19 (5), 452-458.

Park, J., Nakamura, Y., Kwon, Y., Park, H., Kim, E., Park, S., 2010. The effect of combined exercise training on carotid artery structure and function, and vascular endothelial growth factor (VEGF) in obese older women. Jap. J. Phys. Fitness Sports Med. 59 (5), 495-504.

Park, C.L., Riley, K.E., Bedesin, E., Stewart, V.M., 2014. Why practice yoga? Practitioners' motivations for adopting and maintaining yoga practice. J. Health Psychol.

Patel, N.K., Newstead, A.H., Ferrer, R.L., 2012. The effects of yoga on physical functioning and health related quality of life in older adults: a systematic review and metaanalysis. J. Altern. Complement. Med. 18 (10), 902-917.

Raghuram, N., Parachuri, V.R., Swarnagowri, M.V., et al., 2014. Yoga based cardiac rehabilitation after coronary artery bypass surgery: one-year results on LVEF, lipid profile and psychological states-a randomized controlled study. Indian Heart J. 66 (5), 490-502.

Rahnama, N., Namazizadeh, M., Etemadifar, M., Bambaeichi, E., Arbabzadeh, S., Sadeghipour, H.R., 2011. Effects of yoga on depression in women with multiple sclerosis. J. Isfahan Med. Sch. 29 (136)http://onlinelibrary.wiley.com/o/cochrane/ clcentral/articles/048/CN-01016048/frame.html.

Raj, N., Chatterjee, A., Saha, M., 2011. Effect of yoga on diabetes mellitus: a therapeutic approach. Ind. J. Physiol. Pharmacol. 55, 63-64 5 suppl. 1.

Rajajeyakumar, M., Bhattacharjee, M., Amudharaj, D., Madanmohan, Balachander J., 2014. Short term practice of Kapalabhathi pranayam on measurement of human cognitive processing ability in healthy young volunteers. Biomed. 34 (1), 76-81.

Rakhshani, A., Nagarathna, R., Mhaskar, R., Mhaskar, A., Thomas, A., Gunasheela, S., 2015. Effects of yoga on utero-fetal-placental circulation in high-risk pregnancy: a randomized controlled trial. Adv. Prev. Med. 2015, 373041.

Ramen, S., Tripathy, T.B., Mallika, K.J., Shivakumar, MB, Kavita, 2013. A comparative clinical evaluation of ayurvedic diet plan and standard diet plan in sthaulya (obesity). Int. J. Res. Ayurveda Pharm. 4 (5), 680-684.

Ramos-Jimenez, A., Hernandez-Torres, R.P., Wall-Medrano, A., Munoz-Daw, M., TorresDuran, P.V., Juarez-Oropeza, M.A., 2009. Cardiovascular and metabolic effects of intensive Hatha Yoga training in middle-aged and older women from northern Mexico. Int J Yoga. 2 (2), 49-54.

Ray, U.S., Sinha, B., Tomer, O.S., Pathak, A., Dasgupta, T., Selvamurthy, W., 2001. Aerobic capacity \& perceived exertion after practice of Hatha yogic exercises. Indian J. Med. Res. 114, 215-221.

Ray, U.S., Pathak, A., Tomer, O.S., 2011. Hatha yoga practices: energy expenditure, respiratory changes and intensity of exercise. Evid. Based Complement. Alternat. Med. 2011, 241294. 
Rioux, J.G., Ritenbaugh, C., 2013. Narrative review of yoga intervention clinical trials including weight-related outcomes. Altern. Ther. Health Med. 19 (3), 32-46.

Sabet Sarvestani, R., Jamalfard, M.H., Kargar, M., Kaveh, M.H., Tabatabaee, H.R., 2009. Effect of dietary behaviour modification on anthropometric indices and eating behaviour in obese adolescent girls. J. Adv. Nurs. 65 (8), 1670-1675.

Sakuma, Y., Sasaki-Otomaru, A., Ishida, S., et al., 2012. Effect of a home-based simple yoga program in child-care workers: a randomized controlled trial. J. Altern. Complement. Med. 18 (8), 769-776.

Santaella, D.F., Devesa, C.R., Rojo, M.R., et al., 2011. Yoga respiratory training improves respiratory function and cardiac sympathovagal balance in elderly subjects: a randomised controlled trial. BMJ Open. 1 (1), e000085.

Santhi Sri, K.V., Kasturi, K., Sivannarayana, G., 2014. Impact of Pranayama and Amla, an approach towards the control of Diabetes mellitus. Int. J. Pharm. Tech. Res. 6 (3), 1157-1161.

Sarvottam, K., Magan, D., Yadav, R.K., Mehta, N., Mahapatra, S.C., 2013. Adiponectin, interleukin-6, and cardiovascular disease risk factors are modified by a short-term yoga-based lifestyle intervention in overweight and obese men. J. Altern. Complement. Med. 19 (5), 397-402.

Satyanarayana, M., Rajeswari, K.R., Rani, N.J., Krishna, C.S., Rao, P.V., 1992. Effect of Santhi Kriya on certain psychophysiological parameters: a preliminary study. Indian J. Physiol. Pharmacol. 36 (2), 88-92.

Schmidt, T., Wijga, A., Von Zur, Muhlen A., Brabant, G., Wagner, T.O.F., 1997. Changes in cardiovascular risk factors and hormones during a comprehensive residential three month kriya yoga training and vegetarian nutrition. Acta. Physiol. Scand, Suppl. 161 (640), 158-162.

Schulz, K.F., Altman, D.G., Moher, D., 2010. CONSORT 2010 statement: updated guidelines for reporting parallel group randomized trials. Ann. Intern. Med. 152 (11), 726-732.

Shantakumari, N., Sequeira, S., Deeb, R., 2013. Effects of a yoga intervention on lipid profiles of diabetes patients with dyslipidemia. Indian Heart J. 65 (2), 127-131.

Sharma VK, Rajajeyakumar M, Sabramaniam V, et al. Effect of fast and slow pranayama practice on cognitive functions in healthy volunteers. J. Clin. Diagn. Res. 2014;8(1): 10-3.

Sharma, S.K., Yadav, A., Telles, S., Balkrishna, A., 2013. A randomised control trial on yoga and walking for anthropometric and biochemical measures in obese persons. J. Diabetes. 5, 194.

Sharma, N.K., Robbins, K., Wagner, K., Colgrove, Y.M., 2015. A randomized controlled pilot study of the therapeutic effects of yoga in people with Parkinson's disease. Int. J. Yoga. 8 (1), 74-79.

Seo, D.Y., Lee, S., Figueroa, A., et al., 2012. Yoga training improves metabolic parameters in obese boys. Kor. J. Physiol. Pharmacol. 16 (3), 175-180.

Singh, S., Kyizom, T., Singh, K.P., Tandon, O.P., Madhu, S.V., 2008. Influence of pranayamas and yoga-asanas on serum insulin, blood glucose and lipid profile in type 2 diabetes. Indian J. Clin. Biochem. 23 (4), 365-368.

Singh, N., Telles, S., Bhardwaj, A.K., 2011. Effect of yoga and physical exercise on the physical fitness of school children. Indian. J. Physiol. Pharmacol. 55, 45-46 5 suppl. 1.

Sivasankaran, S., Pollard-Quintner, S., Sachdeva, R., Pugeda, J., Hoq, S.M., Zarich, S.W. 2006. The effect of a six-week program of yoga and meditation on brachial artery reactivity: do psychosocial interventions affect vascular tone? Clin. Cardiol. 29 (9), 393-398.

Shukla, R., Gehlot, S., 2014. Response of Paschimottanasana and Kapalabhati on anthropometric parameters in obese individuals as per Prakriti. Int. J. Res. Ayurveda Pharm. 5 (3), 256-260.

Skoro-Kondza, L., Tai, S.S., Gadelrab, R., Drincevic, D., Greenhalgh, T., 2009. Community based yoga classes for type 2 diabetes: an exploratory randomised controlled trial. BMC Health Serv. Res. 9, 33.

Sprod, L.K., Fernandez, I.D., Janelsins, M.C., et al., 2015. Effects of yoga on cancer-related fatigue and global side-effect burden in older cancer survivors. J. Geriat. Oncol. 6 (1), 8-14.

Stachenfeld, N.S., Mack, G.W., DiPietro, L., Morocco, T.S., Jozsi, A.C., Nadel, E.R., 1998. Regulation of blood volume during training in post-menopausal women. Med. Sci. Sports Exerc. 30 (1), 92-98.
Stein, K.M., Weinberg, J., Sherman, K.J., Lemaster, C.M., Saper, R., 2014. Participant characteristics associated with symptomatic improvement from yoga for chronic low back pain. J. Yoga Phys. Ther. 4 (1), 151.

Sukhsohale, N.D., Phatak, M.S., 2012. Effect of short-term and long-term Brahmakumaris Raja Yoga meditation on physiological variables. Indian J. Physiol. Pharmacol. 56 (4), 388-392.

Telles, S., Nagarathna, R., Nagendra, H.R., Desiraju, T., 1993. Physiological changes in sports teachers following 3 months of training in Yoga. Indian J. Med. Sci. 47 (10), 235-238.

Telles, S., Naveen, V.K., Balkrishna, A., Kumar, S., 2010. Short term health impact of a yoga and diet change program on obesity. Med. Sci. Monit. 16 (1), CR35-CR40.

Telles, S., Sharma, S.K., Yadav, A., Singh, N., Balkrishna, A., 2014. A comparative controlled trial comparing the effects of yoga and walking for overweight and obese adults. Med. Sci. Monit. 20, 894-904.

Telles, S., Singh, N., Bhardwaj, A.K., Kumar, A., Balkrishna, A., 2013. Effect of yoga or physical exercise on physical, cognitive and emotional measures in children: a randomized controlled trial. Child Adolesc. Psych. Ment. Health. 7 (1), 37.

Thiyagarajan, R., Pal, P., Pal, G.K., et al., 2015. Additional benefit of yoga to standard lifestyle modification on blood pressure in prehypertensive subjects: a randomized controlled study. Hypertens. Res. 38 (1), 48-55.

Thomley, B.S., Ray, S.H., Cha, S.S., Bauer, B.A., 2011. Effects of a brief, comprehensive, yogabased program on quality of life and biometric measures in an employee population: a pilot study. Explore (NY) 7 (1), 27-29.

Tracy, B.L., Hart, C.E., 2013. Bikram yoga training and physical fitness in healthy young adults. J Strength Cond Res. 27 (3), 822-830.

Trivedi, V., Mishra, P., 2014. The efficacy of classical indian yoga in the treatment of fibromyalgia: a randomized controlled trial. Ann. Rheum. Dis. 73 (Suppl. 2), 295-296.

Tyagi, A., Cohen, M., Reece, J., Telles, S., 2014. An explorative study of metabolic responses to mental stress and yoga practices in yoga practitioners, non-yoga practitioners and individuals with metabolic syndrome. BMC Complement Altern Med. 14, 445.

Van Puymbroeck, M., Payne, L.L., Hsieh, P.C., 2007. A phase I feasibility study of yoga on the physical health and coping of informal caregivers. Evid. Based Complement. Alternat. Med. 4 (4), 519-529.

Villien, F., Yu, M., Barthelemy, P., Jammes, Y., 2005. Training to yoga respiration selectively increases respiratory sensation in healthy man. Respir Physiol Neurobiol. 146 (1) 85-96.

Visweswaraiah, N.K., Telles, S., 2004. Randomized trial of yoga as a complementary therapy for pulmonary tuberculosis. Respirology 9 (1), 96-101.

World Health Organization, 2014. Global status report on noncommunicable diseases 2014. WHO Press, Geneva.

Yadav, R.K., Magan, D., Mehta, N., Sharma, R., Mahapatra, S.C., 2012. Efficacy of a shortterm yoga-based lifestyle intervention in reducing stress and inflammation: preliminary results. J. Altern. Complement. Med. 18 (7), 662-667.

Yadav, R.K., Magan, D., Yadav, R., Sarvottam, K., Netam, R., 2014. High-density lipoprotein cholesterol increases following a short-term yoga-based lifestyle intervention: a nonpharmacological modulation. Acta Cardiol. 69 (5), 543-549.

Yang, K., 2007. A review of yoga programs for four leading risk factors of chronic diseases. Evid. Based Complement. Alternat. Med. 4 (4), 487-491.

Yang, K., Bernardo, L.M., Sereika, S.M., Conroy, M.B., Balk, J., Burke, L.E., 2011. Utilization of 3-month yoga program for adults at high risk for type 2 diabetes: a pilot study. Evid. Based Complement. Alternat. Med. 2011, 257891.

Yang, K., James, K.A., 2014. Yoga, as a transitional platform to more active lifestyle: a 6 month pilot study in the USA. Health Promot Int. Epub ahead of print. doi: 10.1093/heapro/dau108.

Yurtkuran, M., Alp, A., Yurtkuran, M., Dilek, K., 2007. A modified yoga-based exercise program in hemodialysis patients: a randomized controlled study. Complement Ther. Med. 15 (3), 164-171.

Ziv, A., Vogel, O., Keret, D., et al., 2013. Comprehensive approach to lower blood pressure (CALM-BP): a randomized controlled trial of a multifactorial lifestyle intervention. J. Hum. Hypertens. 27 (10), 594-600. 